\title{
Large-scale GPU based DEM modeling of mixing using irregularly shaped particles
}

Nicolin Govender ${ }^{1,2,4}$, Daniel N. Wilke ${ }^{3}$, Chuan-Yu Wu ${ }^{2}$, Raj Rajamani ${ }^{4}$, Johannes Khinast ${ }^{1}$, Benjamin J. Glasser ${ }^{5}$

${ }^{1}$ Research Center Pharmaceutical Engineering, GmbH, Graz, Austria, nicolin.govender@rcpe.at

${ }^{2}$ Department of Chemical Engineering, University of Surrey, United Kingdom

${ }^{3}$ Department of Mechanical and Aeronautical Engineering, University of Pretoria, South Africa

${ }^{4}$ Department of Metallurgical Engineering, University of Utah, Salt Lake City, USA

${ }^{5}$ Chemical and Biochemical Engineering, Rutgers - The State University of New Jersey, USA

\begin{abstract}
Mixing of particulate systems is an important process to achieve uniformity, in particular pharmaceutical processes that requires the same amount of active ingredient per tablet. Several mixing processes exist, this study is concerned with mechanical mixing of crystalline particles using a four-blade mixer. Although numerical investigations of mixing using four-blades have been conducted, the simplification of particle shape to spherical or rounded superquadric particle systems is universal across these studies. Consequently. we quantify the effect of particle shape, that include round shapes and sharp edged polyhedral shapes, on the mixing kinematics (Lacey Mixing Index bounded by 0 and 1) that include radial and axial mixing as well as the inter-particle force chain network in a numerical study. We consider six 100000 particles systems that include spheres, cubes, scaled hexagonal prism, bilunabirotunda, truncated tetrahedra, and a mixed particle system. This is in addition to two six million particle systems consisting of sphere and truncated tetrahedra particles that we can simulate within a realistic time frame due to GPU computing. We found that spherical particles mixed the fastest with Lacey mixing indices of up to 0.9 , while polyhedral shaped particle systems
\end{abstract}


mixing indexes varied between 0.65 and 0.87 , for the same mixing times. In general, to obtain a similar mixing index (of 0.7), polyhedral shaped particle systems needed to be mixed for $50 \%$ longer than a spherical particle system which is concerning given the predominant use of spherical particles in mixing studies.

\section{Introduction}

Granular media is only second to water as the most manipulated substances on the planet [1]. Mixing forms part of the manipulation of granular media, which is essential in a number of industrial processes including civil engineering, pharmaceutical industry and food processing. Mixing is any process designed to combine two or more dissimilar granular materials uniformly to obtain a homogeneous product. The homogeneity of the final product depends on both material properties such as particle sizes (particle size polydispersity), particle shape, moisture content, the mixing method and mixing device. Particle size, volume, density and shape variations may result in demixing (segregation) occurring by percolation, flotation, elutriation, agglomeration, flow-induced or transport mechanisms [2,3]. Segregation may be highly sensitive to particle properties such as flow-induced segregation that is due to small differences in particle size or density when particles flow. Segregation can drastically influence the final product safety and quality.

A number of computational approaches have been developed $[4,5,6,7]$ to predict particle mixing that include isolated cases of continuum models [8] and multi-scale continuum models [9], while the majority of studies considered discrete element models $[10,11,12,13,14,15,16,17,18,19]$. DEM has been shown to be capable to predict sensitive particle scale effects in mixing [10]. Discrete element particle mixing studies have been confined to the modelling of spherical particles $[10,11,12,13,14,15,16,20]$, although the importance of particle shape and angularity from experimental [21, 22] and discrete element studies [10] have highlighted the importance thereof. Laurent and Cleary [23] showed that approximating particles as spherical in a plough mixer under-predicted the free surface angles, leading to lower shear resistance. Cleary et al. [10] demonstrated qualitative agreement between flow patterns but mixing rates were poorly predicted, in particular for lower fill levels which were most likely due to the higher shear resistance of angular particles. This is highlighted by the excerpt and critical observation from Cleary et al. [10] made twenty years ago in 1998, "The most likely cause is in neglecting the real shape of the particles simulation". Hence the validity of studies [24, 25, 26], remain conditional.

Although numerous particle systems are representative of crystalline particles as shown in Figure 1(a), 
only limited DEM studies have considered particle shape in mixing studies. Modifications to spherical particle properties are common to account for the effect of shape, for example, by adjusting rolling friction to account for a mild departure from sphericity [27]. This approach is limited as the relationship of rolling friction to particle shape is usually unknown requiring extensive characterization that diminishes the predictive ability of these models. Studies that considered particle shape usually limit themselves to ellipsoids [28], multi-sphere approximations [29], as depicted in 1(b), or smooth super-quadric particle representations [30, 31, 32], as depicted in Figure 1(c). Polyhedral shape representation that captures the particle angularity as depicted in Figure 1(d), has yet to be conducted for mixing studies.

One of the largest DEM simulation of a mixing device used 8 million spherical particles Radeke et al. [33], which is still far from the billions to trillions of particles required for the simulation of industrially relevant mixing devices. Recent advances in software and computing architectures have realized the utilization of the Graphics Processing Units (GPUs) to solve DEM models. This has resulted in simulations of up to 50 million particles on a single computer [34], and over a billion particles using up to 256 GPUs [35]. However, these large scale simulations drastically oversimplify particle shape by modeling only spheres with basic contact models due to the computational demands of DEM.

Even for smaller scale simulations only a limited number of investigations [31, 32] have considered nonspherical particle shapes. In these studies, non-spherical particle shapes are restricted to super-quadric shapes [31, 32] (see Figure 1(c)) or multi-sphere shapes [36, 37] (see Figure 1(b)). The multi-sphere approach approximates a non-spherical particle using fused spheres [29], as depicted in Figures 1(b). Although the multi-sphere approximation is an improved description for complex particle shapes and allows for particle fragmentation to be taken into account [38], it is limited in the number of spheres that can be used to represent complex particle shapes. It is also unable to accurately capture the particle angularity that may have a significant effect on the behavior of a particulate system. Both the spherical and multi-spherical simplifications significantly reduce the complexity of contact detection that results in significant computational savings. In addition, constitutive models for interacting spheres have been well established and as a result, have been used extensively [39, 40]. Although super-quadric particles allow for improved particle shape representations over the multi-sphere approach they still lack proper angular representation of particles.

Due to the computational demand, polyhedral shaped particles have been limited in discrete element simulations, in particular, when considering proper constitutive models that rely on the overlap volume [41] as opposed to penetration distance [42]. Computing using the overlap volume for polyhedral shaped particles has 
been demonstrated to be computationally tractable [43] on graphical processing units (GPUs). This development is allowing for polyhedral shaped particles to be considered for industrial scale investigations.

This study therefore extends the envelope of discrete element simulations in mixing applications to include the effect of particle angularity and quantify the importance thereof. This is the first study to consider polyhedral particle shape representations that accounts for particle angularity in a mixing study.

\section{BlazeDEM-GPU Simulation Framework}

In this study we use BlazeDEM-GPU developed by Govender et al. [44] that solves Newton's equations of motion for soft particles using an explicit forward Euler time integration scheme on GPU architectures. In a BlazeDEM-GPU simulations, particles are confined by geometric boundaries that are denoted "world objects" to confine particle flow. The world objects are represented as closed-form expressions for curved or planar surfaces. Since, particle shapes can be either spherical or polyhedral that can interact with each other or world objects, requires contact detection to be split into particle-particle contact and particle-world. Recently, Govender et al. [43] demonstrated computational tractable simulations using spherical and polyhedral shaped particles on graphical processing units (GPUs), using the overlap volume to estimate contact force directions and magnitude.

Contact detection is computationally the most expensive part of a DEM simulation accounting for as much as $90 \%$ of the total simulation time for polyhedral shaped particles. Thus importance is placed on implementing algorithms to take full advantage of the parallel nature of the GPU. In addition, algorithms need to utilize memory correctly to minimize the number of memory transactions; otherwise the actual number of threads executing in parallel will be significantly reduced due to register pressure [45]. This is termed the occupancy of a particular method (kernel) executing on the GPU. While a detailed discussion is beyond the scope of this paper, a brief overview on how to maximize the occupancy of the GPU is presented.

Following Govender et al. [34], spheres only require a neighbor search while polyhedra require the neighbour search followed by a detailed particle contact search. Note that typically the particle-particle contact takes up most of the time; we also split the particle-world contact between different kernels to minimize divergence and reduce the number of registers used by the kernel. This can also be run on different GPUs if the geometry is complicated enough to warrant this. The contact detection is followed by contact modeling in which the forces are resolved. Details on the spherical particle-particle contact models have been previously discussed [46, 34]; 
however some of the details of the polyhedral particle-particle contact models has been updated since [34, 44]. In particular, the details on how to resolve the contact force have been updated and validated for hopper flow [43], which we outline in the next section for completeness.

\subsection{Polyhedral particle contact modelling}

The initial BlazeDEM-GPU architecture contact models resolved contact forces and directions purely based on penetration depth [34]. The contact strain energy therefore varied only with penetration depth independent of the volume of material involved during contact $[46,34]$. This assumption proved adequate for low density flows but unsuitable for dense packed systems, in particular when particles interact near edges of world objects, e.g., when particles interact with the lifter edges of ball mills. Isolated particles often exploded off the edges resulting in localised instability in the particle system, as is evident from the BlazeDEM-GPU code that has been made publicly available [44]. This simplistic contact model with its stability concerns, as well as inconsistent contact resolution when contact was resolved close to the edges and vertices, required the inclusion of a volume based contact model. In 2016, BlazeDEM-GPU included a volume based contact model that exactly resolves the contact volume, i.e., the volume common to the polyhedra in contact, and contact normal's as depicted in Figures 2 (a) - (d). The contact volume allows for a better description of the work done during contact [47].

Firstly, the broad phase contact is handled using a bounding sphere approach. Once potential contact has been established the detailed contact is resolved. Once the vertices that define the convex contact volume is computed, the surface integral of the surface normal's of one particle inside the other particles and vice versa establishes the respective reactions normal's as depicted in Figure 2 (c).

Normal Contact The contact volume has a centroid through which the contact normal's are assumed to act, while the contact force magnitude is assumed to be proportional to the contact volume with the volumetric spring stiffness $K_{n}$ being the proportionality constant. Specifically, a Kelvin-Voigt linear viscoelastic spring dashpot for rigid (hard) particles is considered. This results in an elastic force that stores energy and a dissipative Coulomb force that dissipates energy given by

$$
\mathbf{F}_{\mathrm{n}}=\left(\mathrm{K}_{\mathrm{n}} \Delta V\right) \mathbf{n}-\mathrm{C}_{\mathrm{n}}\left(\mathbf{V}_{\mathrm{R}} \cdot \mathbf{n}\right) \mathbf{n},
$$


where $\mathrm{K}_{n}$ is the spring stiffness $\left(\frac{N}{m^{3}}\right), \mathbf{n}$ the normal direction along which the force acts, $C_{n}$ the damping coefficient $\left(\frac{N s}{m}\right)$ where and $\mathbf{V}_{\mathbf{R}}$ the relative velocity between the contacting particles, $\mathrm{K}_{\mathrm{n}}=\frac{\mathrm{m}_{\text {eff }}}{\mathrm{t}_{\text {contact }}^{2}} \ln (\epsilon)^{2}+\pi^{2}$ is the spring stiffness, $\mathrm{C}_{\mathrm{n}}=\frac{2 \ln (\epsilon) \sqrt{\mathrm{K}_{\mathrm{n}} \mathrm{m}_{\text {eff }}}}{\sqrt{\ln (\epsilon)^{2}+\pi^{2}}}$ is the viscous damping coefficient, $\overline{\mathbf{n}}$ the normal at contact, $\epsilon$ is the coefficient of restitution and $\mathrm{m}_{\mathrm{eff}}=\left(\frac{1}{\mathrm{~m}_{1}}+\frac{1}{\mathrm{~m}_{2}}\right)^{-1}$ is the effective mass of the particles. The contact time $t_{\text {contact }}$ is determined by the properties of the material and is chosen such that physical quantities of interest (such as energy) are conserved during integration for the typical range of velocities observed in the simulations $[48,49,50]$. For all simulations in this study we use a contact time equivalent to at-least 10 time steps. The overlap volume $\Delta V$ and contact normal are resolved exactly for two polyhedral shaped particles in contact as depicted in Figures 2 (a) - (d), while for spheres it is just the relative overlap.

Tangent Contact Tangential contact between particles are resolved using a stick-slip friction model where the tangential force is coupled to the normal force through Coulomb's law. The initial tangential force is computed as the sum of the tangential spring force and a tangential viscous force

$$
\mathbf{F}_{\mathrm{T}}=-\mathrm{K}_{\mathrm{T}}\left(\mathbf{V}_{T} \mathrm{dt}+\mathbf{L}\right)-\mathrm{C}_{\mathrm{T}} \mathbf{V}_{\mathrm{T}}
$$

where $\mathbf{L}$ is the tangential spring displacement from its equilibrium position, $K_{T}$ the tangential spring stiffness and is typically set to be at least $\frac{1}{2} \mathrm{~K}_{n}$, and $\mathrm{C}_{\mathrm{T}}=\frac{2 \ln (\epsilon) \sqrt{\mathrm{K}_{\mathrm{T}} \mathrm{m}_{\text {eff }}}}{\sqrt{\ln (\epsilon)^{2}+\pi^{2}}}$ the tangential damping coefficient, and $\mathbf{V}_{\mathrm{T}}$ the relative tangential velocity. For the static friction case where $\mathbf{f}_{T}^{0}$ is below the Coulomb limit, the tangential spring magnitude is incremented by L given by

$$
\mathbf{L}=\int_{\mathbf{t}_{\text {Cstart }}}^{\mathbf{t}_{\text {Cend }}} \mathbf{v}_{\mathbf{T}} d t
$$

where the left-endpoint numerical integration is conducted over every time step, whereas for sliding friction $\mathbf{f}_{T}=\mathbf{f}_{T}^{0}$. Hence, the number of simulation time steps over which the integration is effectively computed is given by $\frac{t_{\text {Cend }}-t_{C \text { start }}}{\Delta t}$.

Angular Motion In addition to translation forces a particle also experiences a torque as a result of contact given by : 


$$
\boldsymbol{\Gamma}=\left(\mathbf{r} \times \mathbf{F}_{\mathbf{n}}\right)
$$

where $\mathbf{r}$ is the vector from the COM to the contact point $\mathbf{P C}(x, y, z)$. The angular velocity $\omega$ of particle $i$ at time $\mathrm{k}$ is obtained using the forward Euler integration scheme.

$$
\boldsymbol{\omega}_{\mathrm{k}}=\boldsymbol{\omega}_{\mathrm{k}-1}+\boldsymbol{\alpha}_{\mathrm{k}}^{\mathrm{ang}} \triangle \mathrm{t}
$$

The angular acceleration $\boldsymbol{\alpha}^{\text {ang }}$ at time k is given by $\boldsymbol{\alpha}_{\mathrm{k}}^{\mathrm{ang}}=\mathbf{I}_{\mathrm{k}}^{-1} \boldsymbol{\Gamma}_{\mathrm{k}}^{\mathrm{net}}$ where $\boldsymbol{\Gamma}_{\mathrm{k}}^{\mathrm{net}}=\sum_{j=1}^{L} \boldsymbol{\Gamma}^{\mathrm{ij}}$ is the sum of all $L$ body contact torques experienced by particle $i$ as given in Equation 4 and $\mathbf{I}_{\mathrm{k}}$ the inertia tensor at time $\mathrm{k}$. Quaternions have minimal storage requirements and are thus well suited to the GPU, and they are also more robust than other representations such as Euler angles [51]. The orientation of a particle is represented by a unit quaternion $\mathbf{q}\{\mathrm{w}, \mathrm{x}, \mathrm{y}, \mathrm{z}\}=\{1,0,0,0\}$, where $\mathrm{w}$ is an angle $[-1: 1]$ and $(\mathrm{x}, \mathrm{y}, \mathrm{z})$ the axis of rotation. The relationship between a quaternion and axis angle representation $\left(\theta, x_{1}, y_{1}, z_{1}\right)$ is given by:

$$
\mathbf{q}=\left\{\cos (\theta / 2), x_{1} \sin (\theta / 2), y_{1} \sin (\theta / 2), z_{1} \sin (\theta / 2)\right\}
$$

Given an angular velocity vector $\boldsymbol{\omega}$ the quaternion representing that rotation is given by:

$$
\triangle \mathbf{q}=\left\{\cos \left(\left\|\boldsymbol{\omega}_{\mathrm{k}}\right\|\right), \sin \left(\left\|\boldsymbol{\omega}_{\mathrm{k}}\right\| \frac{\boldsymbol{\omega}_{\mathrm{k}}}{\left\|\boldsymbol{\omega}_{\mathrm{k}}\right\|}\right)\right\}
$$

The evolution of the angular orientation of the particle is just a multiplication [52] between the current quaternion $\mathbf{q}_{\mathrm{k}-1}$ of a particle with $\triangle \mathbf{q}$ :

$$
\mathbf{q}_{\mathrm{k}}=\mathbf{q}_{\mathrm{k}-1} \times \triangle \mathbf{q}
$$

\section{Simulation Model Setup}

In this study we investigate the effect of particle shape on the mixing effectiveness of a high-shear mixer for batch processing using different particle shapes. We now outline the simulation model setup that considers the 
mixing device, particle shapes and required data processing. We use BlazeDEM-GPU [44] developed by the authors, which has been validated for a number of applications [46, 53, 54]. BlazeDEM-GPU is being used by a growing number of users in the Americas, Europe, Africa, Asia and Australia.

\subsection{Mixing Device}

In this study we investigate the effect of particle shape on the mixing effectiveness of a high-shear mixer for batch processing for different particle shapes. We consider a $1.0 \mathrm{~m}$ diameter high-shear batch mixer with four blades pitched at 45 degrees rotating inside a stationary vessel as outlined in Figure 3. The mixer operates at $22 \mathrm{rpm}$ in the anti-clockwise direction. Particles are filled from a height of $28 \mathrm{~cm}$ under gravity deposition resulting in an initial particle bed that is just covering the top of the blades. This high-shear batch mixer is typically used for materials that tend to segregate or agglomerate and as a consequence is not suitable to be mixed in tumbling mixers. This mixer has been used in a number of experimental and numerical studies in the pharmaceutical and chemical industries $[55,56,32]$. The outer cylinder and inside rotor are both modeled analytically as cylinders, hence these world objects are modelled as round instead of triangulated as faces which is often done. In addition, each of the four blades is modelled by faces, edges and vertices to exactly represent four rectangular prisms.

\subsection{Particles}

We will be considering non-symmetric sharp edge particles of homogeneous and non-homogeneous mixtures, which is distinct from the superquadrics considered by Clearly et.al [31]. In particular, the particles considered in this study are depicted in Figure ??, where particles are restricted to the same volume. Polyhedral shaped particles are modeled with sharp edges as opposed to the clumped sphere and superquadric approximations. Table 1 lists the geometric and mass properties for the various particles used, including, bounding radius (Bound Radius), volume, aspect ratio, mass and surface area (SArea). The aspect ratios of the particles varied between 1 and 6.68 where the aspect ratio is defined as the ratio between the extreme maximum and minimum extent from the Cartesian directions. Note all the particles had similar masses within $3 \%$.

In this study, the following parameters are used for all particles such that numerical stability is achieved :

1. normal stiffness: $1.56 \times 10^{6} \frac{\mathrm{N}}{\mathrm{m}}$,

2. tangential stiffness: $0.697 \times 10^{6} \frac{\mathrm{N}}{\mathrm{m}}$, 
3. coefficient of restitution: 0.45 ,

4. normal damping: 26.85 ,

5. tangential damping: 17.09 ,

6. coefficient of friction: 0.50 ,

7. rolling resistance (spheres only): 0.01

Note that the contact volume is converted to contact distance by taking the cubic root of the volume before multiplying it with the normal or tangential stiffness. In addition, all simulations are conducted using a time step of $\triangle t=1 \times 10^{-5}$ s, i.e., 100000 time steps per second of process time. The stiffness parameters are chosen such to ensure at least 10 time steps during loading and unloading.

\subsection{Statistical Analysis}

For all simulations the total mass of particles is equally divided into two species with identical mechanical properties and particle shapes for each species. Each species is assigned a different color to serve as an identifier allowing for qualitative evaluation of mixing. Mixing is quantified using the Lacey Mixing Index [57] (bounded by 0 and 1) for binary mixtures, in which first we divide the volume of interest into a cubic grid of $5 \mathrm{~cm}$ by $5 \mathrm{~cm}$ by $5 \mathrm{~cm}$ cells (or voxels). Only cells that include particles are retained to give $N=2400$ for $n$ particles. Let $\bar{x}=\frac{1}{N} \sum_{i=1}^{N} x_{i}$, be the mean concentration for a reference component and $x_{i}$ the concentration in the $i^{\text {th }}$ cell of the reference component. The variance $\sigma^{2}$ of the concentration for the reference component is given by

$$
\sigma^{2}=\sum_{i=1}^{N} \frac{x_{i}-\bar{x}^{2}}{N-1},
$$

where the mean concentration for a reference component is estimated from $\bar{x}=\frac{1}{N} \sum_{i=1}^{N} x_{i}$. For binary mixtures of fractions $p$ and $(1-p)$ the variance of a random mixture (minimum variance) is given by

$$
\sigma_{R}^{2}=\frac{p(1-p)}{n},
$$

while the variance of a completely segregated mixture (maximum variance) is given by

$$
\sigma_{0}^{2}=p(1-p)
$$


which allows us to express the Lacey Mixing Index (M) for the sample as

$$
M=\frac{\sigma_{0}^{2}-\sigma^{2}}{\sigma_{0}^{2}-\sigma_{R}^{2}}
$$

In this study the Lacey Mixing Index is resolved for each particle system using $\mathrm{N}$ samples with an average of 42 particles per sample.

\subsection{Spatial Statistical Analysis}

In addition, to the Lacey Mixing Index we compute the Relative Standard Deviation (RSD) which has been pointed out to better reflect the state of mixing and is preferred in industry [55]. The RSD is then computed as the standard deviation $\sigma$ divided by the mean concentration $\bar{x}$ as stated by

$$
R S D=\frac{\sigma}{\bar{x}}
$$

In this study RSD is resolved spatially by computing RSD for each $i^{\text {th }}$ voxel, which is sampled to compute the standard deviation $\sigma$ and sample concentration $x_{i}$ for the voxel, while a reference concentration of $\bar{x}=0.5$ is used. An RSD of 0 implies a uniform sample, i.e., the lower the RSD the more homogeneous is the mixture. In this study the mixing index for all simulations ran until variations in the mixing index became negligible, which occurred after 30s of simulation time. Although this analysis is not currently possible experimentally it does provide a detailed understanding of the mixing efficiency of the various shapes for spatial uniformity over the mixing domain.

\section{Numerical mixing study}

We investigate the effect of five particle shapes on mixing by starting with a mixture of mono-disperse particles separated into two color groups as two horizontal layers on top of each other, as depicted in Figure 5. The red particles are filled first following by the green particles with each layer having 50000 particles. In addition, to the five mono-dispersed homogeneous particle systems we also consider one poly-disperse heterogeneous particle system that is made of equal mass fractions of all four polyhedral (Cubes, TTet, Biluna and HexPrism) particles (Mix). The total number of particles of 100000 equates to $144.6 \mathrm{~kg}$ and a particle volume of around 520,000 
$\mathrm{cm}^{3}$.

Figure 6 (a) - (b), respectively depict the Lacey Mixing Index and Smoothed Lacey Mixing Index Rate evolution for the mono-dispersed Cube, Sphere, TTet, Biluna, HexPrism and Mix particulate systems of 100 000 particles for each system. Although we ran all simulations for sixty seconds, the mixing index started to level off significantly after thirty seconds for almost all particle systems.

\subsection{Averaged and Spatial Lacey Mixing Indices}

The Lacey Mixing Index depicted in Figure 6 (a), indicates that the Sphere particle system mixed the fastest, which is consistent with the findings of Cleary et al. [32]. This can be attributed to the spheres ability to flow much easier allowing the top and bottom layers to mix easier. Moreover, the Sphere particle system reached the highest mixing quality after 30s followed by the Cube and then Mix particle systems. Note that the mix particle system initially achieved a higher mixing index than the Cube particle system. It is clear that the TTet and Biluna particle systems achieved the same mixing index although the TTet particle system required less time for the mixing indices to level off. In addition the Mix, TTet and Biluna particle systems were the first to level off. Evidently, from 25s onwards, the Cumulative Lacey Mixing Index tends towards a straight line for each particle system, which indicates either a constant i.e. steady-state or slowly accumulating mixing index. The final mixing index varied between 0.66 (HexPrism) and 0.88 (Sphere). The TTet and Biluna particle systems achieved a Lacey Mixing Index of 0.75 that is $15 \%$ less than the spheres. Not surprising, the HexPrism particle system with the largest aspect ratio was the slowest to mix with a Lacey Mixing Index of 0.66 after 30s, which is $25 \%$ lower than that for the Sphere particle system. In general, to obtain a similar mixing index (of 0.7), most polyhedral shaped particle systems need to be mixed for $50 \%$ longer than a spherical particle system, while the HexPrism particle system is unable to achieve a Lacey Mixing Index of 0.7 for this specific mixing process.

The Smoothed Lacey Mixing Index Rate (SLMIR) is computed by differentiating and smoothing the Lacey Mixing Index (LMI). By direct differentiation of the Lacey Mixing Index, we obtain a rather noisy Lacey Mixing Index Rate, which is smoothed by averaging it over 4 time steps i.e. 1s.

Note that all the polyhedral particle systems including the Mix particle system exhibit a sharply decreasing mixing rate initially $(\mathrm{t}<5 \mathrm{~s})$ followed by an overall moderately decreasing mixing rate between $5 \mathrm{~s}$ and $15 \mathrm{~s}$ as indicated by the SLMIR in Figure 6(b). For the polyhedral particle systems, the overall moderately decreasing mixing rates do however exhibit intermittent increases in mixing rate or plateaus of constant mixing rate as 
is evident by the Biluna and HexPrism particle system from $8 \mathrm{~s}$ to $13 \mathrm{~s}$. However, the Sphere particle system seems to exhibit a more gradual decrease in SMLIR. This again highlights the importance of considering particle shapes accurately and including angularity in mixing simulations.

It is important to note that the differences in Lacey Mixing Indices is due to mixing and not a result of the computation of the Lacey Mixing Index for the various particles systems. This was confirmed by dropping particles randomly into the mixing container for which the Lacey Mixing Index varied only between 0.89 (Sphere particle system) and 0.93 (HexPrisms).

Figure 12 illustrates the spatial distribution of the Relative Standard Deviation (RSD) over the mixing domain at $\mathrm{t}=30 \mathrm{~s}$. It can be seen that the spheres mix in a fairly homogeneous fashion as expected while the hexagonal prisms have in-homogeneous regions around the blade positions. The spatial distribution for the TTet, Biluna and Mix particle systems are very similar, while the Cube particle system is similar to the Sphere particle system which is in agreement with the reported Lacey Mixing Index in Figure 6.

\subsubsection{Statistical significance}

We quantify the statistical significance of the reported mixing from 25 s to 30 s for each particle type, which is depicted in Figure 7. We first report the relative standard deviation (RSD) in Table ??, which indicates a variation between $0.415 \%$ and $1.563 \%$. This is indicative of a tight normal distribution as the standard deviation represents a variation of at most $1.6 \%$ of the sample mean. In addition, by conducting 2 -sample $t$ tests of the mixing indices for the various particle types we quantify the statistical significance between the reported sample means for the various particle types. The 2-sample t tests are reported in Table ??, with each index indicating a [t-value, p-value] pair.

The results demonstrate strong statistical significance between the reported mixing index means as characterized by large t-values, i.e. large differences between the sample distrubtions expressed in units of standard deviation, or extremely small p-values, which is a clear rejection of the null hypothesis. It is clear that all the reported mixing index means are statistically significant w.r.t. each other except for the TTet and Biluna particle systems that largely overlap which is indicative of a low Kullback-Leibler divergence or evidence of high mutual information. 


\subsection{Mixing Kinematics}

Figures 8 and ?? depict the simulation with two colored layers and the resultant speed to show the mixing state and kinematics at $\mathrm{t}=30 \mathrm{~s}$. Note that the polyhedral particle systems are rendered with shading that results in shaded green and shaded red particles, while the resultant speeds were calculated as the $L_{2}$-norm of the translational and rotational velocities. Figure 8 depicts the side view for the various shapes, highlighting the void region behind the blade by the overlaid black solid lines. We see the Mix and Cube particle systems have the largest void region as they pack in a highly stable fashion behind the blade while flowing over the top of the blade at a greater height due to the ordered packing. This causes shear bands that results in higher entropy over the blade to enhance mixing of the layers. In Figure 8, variations in the velocity profiles illustrate that particle shape affects the localization of high and low velocity domains, where the Spheres particle system demonstrates a more gradually changing velocity profile while the polyhedral shaped particles tend to be more localised having a larger spatial variation over the same spatial domain. Again the polyhedral particle systems are rendered with shading that results in shaded velocity colors.

Figures ?? (a) - (f) depict the resultant speed and resultant angular speed, both are the instantaneous speeds at 30s, for the Cube, Spheres, TTet, Biluna, HexPrism and Mix particle systems, respectively in the columns 1-2 and columns 3-4. The resultant speeds were calculated as the $L_{2}$-norm of the translational and rotational velocities. We notice that the higher resultant speeds at the bottom of the mixer for the Sphere and Cube particle systems are more spatially spread out as compared to the other polyhedral particle systems. This occurs because both the Sphere and Cube particle systems at the bottom tend to be packed into ordered and stable packing's, which allows for a better transfer of kinetic energy between particles resulting in higher velocities being more spread out. The extent of the mixing is also evident from large aspect ratio. From the top view we see that the polyhedra have more defined stagnant zones, while rotation of polyhedra is limited compared to the Sphere particle system that have a low rolling resistance, resulting in a wider velocity distribution.

This is further demonstrated for the resultant angular speed depicted in Figure ??. Here, the high angular speed of the Sphere particle system relative to the polyhedral particle systems is as a result of the low rolling resistance between spheres. Of significance for polyhedra is that the angular speed is limited around the blade area with the HexPrism particle system being the most localised and most limited to rotate due to the large aspect ratio between particles. Of interest is the Biluna and Mix particle systems that are also more localised 
when compared to the Cube and TTet particle systems. This implies that rotations in the Biluna and Mix particles systems are a limiting mechanism when compared to Cube and TTet particle systems. As expected, low rolling resistance in spheres implies rotations to be much more prevalent allowing particles to roll off inclines to enhance mixing in the Sphere particle system when compared to a polyhedral particle system. This is confirmed by revisiting the third and fourth column of Figures ?? (a) - (f), which depicts the bottom and top view shaded by resultant angular speed from both the Cube, Spheres, TTet, Biluna, HexPrism and Mix particle systems respectively. We notice that the angular speeds at the bottom of the mixer for the Sphere and Cube particle systems are more spatially spread out as compared to the other polyhedral particle systems. This is because both the Sphere and Cube particle systems pack into a stable lattice that effectively locks the particles in place to allow the kinetic energy of the mixer to be transferred to a larger number of particles, as opposed to shearing that localizes the energy transfer. From the top view it can be seen that the polyhedra have more defined stagnant zones as rotation for polyhedra is limited compared to spheres that have a low rolling resistance, resulting in wider velocity fields. The highly localised rotational zones for the HexPrism particle system around the blades is as expected and confirmed from Figure ?? (e). Interestingly, the spatial areas of high angular speed is localised for most of the polyhedral particulate systems as the particles settle after the blade has passed. It is important to note that the Sphere particle system has high angular velocity over large domains of the mixer bottom. This implies that the particles tend to roll more than slide at the bottom of the blender which requires less energy to rotate the particle bed than what sliding of the particles would require. This in conjunction with the translational velocity at the bottom of the mixer implies that the particles are not purely rolling but rather rolling and slipping, which implies the maximum frictional force to ensure rolling has been exceeded and as a result a varying translational velocity at the bottom of the bed is evident.

\subsection{Mixing Kinetics}

Lastly, we investigate the mixing kinetics by investigating the inter-particle forces. Towards this aim we consider the developed force chains for the various particle systems considered in this study. In particular, we isolate the force chain network developing in front of one mixing blade after 25 seconds. The force chains are normalized between 0 and 8, and only force chains between 0.5 and 8.0 are depicted in Figures 13 (a) - (f). Note the well developed force network of the Cube particle system, depicted in Figure 13 (a), followed by the Sphere, TTet and Mix particle systems respectively depicted in Figures 13 (b), (d) and (f). It is evident that the force 
chains networks for the Hex and Biluna particle systems are finer, this consequently results in fewer strong inter-particle forces. It is evident that larger forces in the regular packed Cube and Sphere particle systems extend further ahead of the mixing blade, while the inter-particle forces for the other particle systems tend to break down in to smaller forces. The force chains along the mixing blade extends the highest for the Cube, TTet and Biluna particle systems, while the Sphere particle system tends to disintegrates after a certain height. This is most likely due to lower relative shear motion between Sphere particles as a result of rolling. It is evident that the inter-particle forces is a strong function of the particle shape, which ultimately relates to differences in mixing and particle degradation during mixing.

The average start-up power, averaged over the first $0.01 \mathrm{~s}$ for the same prescribed rotation rate (rpm is constant), for the various particle systems are also indicated in Figure 12. The power is computed as $\frac{T N}{9.5488} \mathrm{~kW}$, where $\mathrm{T}$ is the torque in N.m and $\mathrm{N}$ the revolutions per minute. The torque is obtained by summing all contact forces with the four blades crossed with the radial distance from the contact point to the center shaft. In essence particle shape may result in a $44 \%$ variation of power consumption for the same mixing process. The Hex particle system had the largest power demand followed by the Cube and Mix particle systems, while the Sphere particle system required the least energy input. This is due to the inter-particle shear resistance, which is the largest for the Hex prism that has the largest aspect ratio, followed by the Cubic particle system that is the most angular. In turn, power dissipation for particle-particle interactions were between $65 \%$ and $72 \%$, particle-blade interactions between $1 \%-2 \%$ and particle-drum interactions in $28 \%$ and $34 \%$ variation. The power dissipation's for particle-particle, particle-blade and particle-drum interactions were calculated by isolating the particle-particle, particle-blade and particle-drum contacts and then computing the dissipated energy for each over the first 0.01s. As expected the lowest start-up power is required by the Sphere particle system, while the highest demand is required for the Hex particle system. The Cube and Mix, and, TTet and Biluna, particle systems have respectively similar power demands, while the Cube and Mix particle systems have higher power. The percentage power demands between particle-particle interactions is the highest for the HexPrism and Sphere particle systems, while the particle-blade interactions is similar between all particle systems. The largest percentage of the power demands for particle-drum interactions is for the Biluna and TTet particle systems. Particles jamming initially in front of the blade is also of interest to note, the jamming may become more or less prevalent at mixing which may cause changes in the power profile from start-up to steady-state. However, as the blade accounts for at most $2 \%$ power dissipation at startup, a maximum upper bound on the power dissipation is around $4 \%$ due to particles jamming in front of the blade. The normal power demand 
at startup for particle-particle interactions is at least $58 \%$ for polyhedral particle systems, while the Sphere particle system only accounted for $37 \%$. The Hex particle system had the largest normal particle-particle power dissipation at startup at $69 \%$.

In addition, to the initial power dissipation we include the normal and shear energy spectra after $25 \mathrm{~s}$ for each particle system normalized w.r.t. Sphere particle system. This spectra is representative of the average normal and shear energy over the first $25 \mathrm{~s}$ of the simulation. The normalized normal and shear energy $(\mathrm{J} / \mathrm{J})$ spectra is given in Figures 14(a) and (b). It is evident the Cube particle system dominates the normal and shear dissipation, while the Biluna particle system has mostly the lowest normal and shear dissipation. Except for the Cube particle system, the normal and shear energy spectra for the other particle systems increase relative to the Sphere particle system for energies above 1E-6, while for energies below $1 \mathrm{E}-6$ they tend to decrease w.r.t. to the Sphere particle system.

In addition, the normalized normal and shear impact (Ns/Ns) damage for each particle accumulated over the mixing duration relative to the Sphere particle system is given in Figures 15 (a) and (b). It is evident that on average all polyhedral systems including the Mix particle system experience larger impacts than the Sphere particle system. This has profound consequences on the particle degradation under normal and shear impacts, highlighting again the importance of particle shape.

\section{$5 \quad$ Large Scale Mixing Simulation}

To conclude the numerical investigation in this study, we consider two large scale mixing simulations. One using 6 million Sphere particles, while the other uses 6 million TTet particles. Figure ?? depicts the initial mixer state with 6 million TTet particles each having a mass of $0.00005003 \mathrm{~kg}$ and volume of $0.017868 \mathrm{~cm}^{3}$ in a left-right split resulting in a total particle mass of $300.18 \mathrm{~kg}$ and packing volume of $107080 \mathrm{~cm}^{3}$. The only difference from the previous simulation is that for this industrial case the blades are $5 \mathrm{~cm}$ shorter and the rotor with four blades is rotating at $30 \mathrm{rpm}$.

Figures ?? (a) - (c) and Figures ?? (d) - (f) depict the system state after 60s for the TTet and Sphere particle systems respectively. We notice that the motion of the blade affects a larger part of the bed than for the smaller scale simulations. We see that beyond the blade tip, the TTet particles remained more segregated, while the Spheres mixed more efficiently. This is due to the spherical particles interacting with particles at the drum wall allowing for mixing, while the polyhedra tend to rotate and pack around the drum to form a stable 
shear plane around the layer of particles around the drum wall. The Mixing Index for the TTet particle system after 15 revolutions was 0.62 , while for the Sphere particle system it was 0.72 . In addition, the Mixing Index for the Sphere particle system is $20 \%$ higher than the TTet particle system, which also illustrates the importance of shape for large scale simulations.

We demonstrated for the 100000 and 6 million particle systems that the influence of particle shape is significant, with the Sphere particle system having a $17 \%$ to $20 \%$ higher mixing index after 30 s than the TTet particle systems in two separate mixing studies. For the 6 million particle system a mixing index of 0.72 was reached after 30s, while this was reached after around 11s for the 100000 particle system. Similarly, the 6 million TTet particle system reached a mixing index of 0.62 after 30s, while the 100000 particle system reached this around 12s. Although the 6 million and 100000 particle system simulations are not directly comparable due to changes in the blade and total particle mass, it is important to note that the observations made on the 100000 particle system do generalize to industrial scale simulations. As outlined, the relative difference in mixing index between the two particle shapes do not diminish with increase in the number of particles and remains an important modeling parameter to capture accurately.

\section{Conclusion}

This study quantified the difference in the Lacey Mixing Index and relative standard deviation of mixing between six particle systems that included spheres, cubes, scaled hexagonal prism, bilunabirotunda and truncated tetrahedra particles. The mixing indices after 30 s varied between 0.66 and 0.88 for the various particle systems with a 10s variation in the levelling, with only the particle shape varying between the particle systems. The importance of accurately modelling angularity and aspect ratio is evident. In addition, the effect of particle shape on the resultant speed and angular speed spatial distributions, and power demands for a high-shear batch blender with four blades has been demonstrated by a numerical investigation. The investigation were extended to include mixing kinetics, that highlighted differences in inter-particle forces by isolating the force chain network in front of a mixing blade after 25s. As demonstrated particle shape may result in a $44 \%$ variation of power consumption for the same mixing process, where the power dissipation for particle-particle interactions varied between $65 \%$ and $72 \%$, particle-blade interactions varied between $1 \%-2 \%$ and particle-drum interactions resulted in a $28 \%-34 \%$ variation. Importantly, for the particle-particle interactions the percentage normal interactions where at least $58 \%$ for the polyhedral particle systems, while for the Sphere particle system it was 
only $37 \%$.

These differences should be considered in the design and operation of high-shear batch blenders. At the same time, these results are for a limited number of cases and further work is needed to confirm that similar results are observed for other particle properties and mixer conditions.

\section{Acknowledgments}

We gratefully acknowledge the support of NVIDIA Corporation with the donation of the Titan X Pascal GPUs used for this research. The financial support of the National Research Foundation of South Africa is acknowledged. This work was supported by the MARIE Sklodowska-CURIE Individual Fellowships with acronym DECRON, funded through the People Programme (MARIE Sklodowska-CURIE Actions) of the European Union's H2020 under REA grant agreement No. 747963.

\section{References}

[1] P. G. de Gennes, Granular matter: a tentative view, Rev. Mod. Phys. 71 (1999) S374-S382. doi:10.1103/RevModPhys.71.S374.

URL https://link.aps.org/doi/10.1103/RevModPhys.71.S374

[2] A. Scott, J. Bridgwater, Interparticle percolation: A fundamental solids mixing mechanism, Ind. Eng. Chem. Fundamen. 14 (1975) 22-27.

[3] S. N. Pathak, V. Esposito, A. Coniglio, M. Ciamarra, Force percolation transition of jammed granular systems, Physics Review E 86 (2017) 042901.

[4] D. Khakhar, A. Orpe, J. Ottino, Continuum model of mixing and size segregation in a rotating cylinder: Concentration-flow coupling and streak formation, Powder Technology 116 (2-3) (2001) 232-245. doi:10.1016/S0032-5910(00)00390-9.

[5] I. Christov, J. Ottino, R. Lueptow, From streamline jumping to strange eigenmodes: Bridging the lagrangian and eulerian pictures of the kinematics of mixing in granular flows, Physics of Fluids 23 (10). doi:10.1063/1.3653280. 
[6] P. K. Haff, Grain flow as a fluid-mechanical phenomenon, Journal of Fluid Mechanics 134 (1983) 401-430.

[7] S. W. Meier, R. M. Lueptow, J. M. Ottino, A dynamical systems approach to mixing and segregation of granular materials in tumblers, Advances in Physics 56 (5) (2007) 757-827. doi:10.1080/00018730701611677. URL https : //doi.org/10.1080/00018730701611677

[8] C. Davalos, J. Cante, J. Hernandez, J. Oliver, On the numerical modeling of granular material flows via the particle finite element method (pfem), International Journal of Solids and Structures 71 (2015) 99 125. doi:https://doi.org/10.1016/j.ijsolstr.2015.06.013.

URL http://www.sciencedirect.com/science/article/pii/S0020768315002772

[9] M. Fang, K. Luo, S. Yang, K. Zhang, J. Fan, Computational Fluid Dynamics-Discrete Element Method Investigation of Solid Mixing Characteristics in an Internally Circulating Fluidized Bed, Industrial \& Engineering Chemistry Research 52 (22) (2013) 7556-7568. doi:10.1021/ie400306m.

URL https ://doi.org/10.1021/ie400306m

[10] P. Cleary, G. Metcalfe, K. Liffman, How well do discrete element granular flow models capture the essentials of mixing processes?, Applied Mathematical Modelling 22 (1998) 995-1008.

[11] M. Sen, S. Karkala, S. Panikar, O. Lyngberg, M. Johnson, A. Marchut, E. Schafer, R. Ramachandran, Analyzing the mixing dynamics of an industrial batch bin blender via discrete element modeling method, Processes $5(2)$.

[12] A. Hassanpour, M. Ghadiri, Discrete Element Method (DEM) Simulation of Powder Mixing Process, John Wiley \& Sons, Ltd, 2015, pp. 459-477. doi:10.1002/9781118682692.ch17.

URL http://dx.doi.org/10.1002/9781118682692.ch17

[13] N. Gui, J. Gao, Z. Ji, Numerical study of mixing and thermal conduction of granular particles in rotating tumblers, AIChE Journal 59 (6) (2013) 1906-1918. doi:10.1002/aic.13999.

URL http://dx.doi.org/10.1002/aic.13999

[14] E. Alizadeh, F. Bertrand, J. Chaouki, Discrete element simulation of particle mixing and segregation in a tetrapodal blender, Computers \& Chemical Engineering 64 (2014) 1 - 12. doi:https://doi.org/10.1016/j.compchemeng.2013.12.009.

URL http://www.sciencedirect.com/science/article/pii/S0098135413003906 
[15] F. Qi, T. J. Heindel, M. M. Wright, Numerical study of particle mixing in a lab-scale screw mixer using the discrete element method, Powder Technology 308 (2017) $334 \quad$ - 345. doi:https://doi.org/10.1016/j.powtec.2016.12.043.

URL http://www.sciencedirect.com/science/article/pii/S003259101630910X

[16] H. Kuo, P. Knight, D. Parker, M. Adams, J. Seville, Discrete element simulations of a high-shear mixer, Advanced Powder Technology 15 (3) (2004) 297 - 309. doi:https://doi.org/10.1163/156855204774150109. URL http://www.sciencedirect.com/science/article/pii/S0921883108601168

[17] B. Alchikh-Sulaiman, M. Alian, F. Ein-Mozaffari, A. Lohi, S. R. Upreti, Using the discrete element method to assess the mixing of polydisperse solid particles in a rotary drum, Particuology 25 (2016) 133 - 142. doi:https://doi.org/10.1016/j.partic.2015.05.006.

URL http://www.sciencedirect.com/science/article/pii/S1674200115001522

[18] G. Basinskas, M. Sakai, Numerical study of the mixing efficiency of a ribbon mixer using the discrete element method, Powder Technology 287 (2016) 380 - 394. doi:https://doi.org/10.1016/j.powtec.2015.10.017.

URL http://www.sciencedirect.com/science/article/pii/S0032591015301066

[19] M. Sakai, Y. Shigeto, G. Basinskas, A. Hosokawa, M. Fuji, Discrete element simulation for the evaluation of solid mixing in an industrial blender, Chemical Engineering Journal 279821 - 839. doi:https://doi.org/10.1016/j.cej.2015.04.130.

URL http://www.sciencedirect.com/science/article/pii/S1385894715006208

[20] M. Alian, F. Ein-Mozaffari, S. R. Upreti, Analysis of the mixing of solid particles in a plowshare mixer via discrete element method (dem), Powder Technology 274 (2015) 77 - 87. doi:https://doi.org/10.1016/j.powtec.2015.01.012.

URL http://www.sciencedirect.com/science/article/pii/S0032591015000224

[21] P. Cleary, M. Sawley, DEM modelling of industrial granular flows: 3D case studies and the effect of particle shape on hopper discharge, Applied Mathematical Modelling 26 (2002) 89-111.

[22] J. Latham, A. Munjiza, The modelling of particle systems with real shapes, Philosophical Transactions of the Royal Society of London, Series A: Mathematical, Physical and Engineering Sciences 362 (2004) $1953-1972$. 
[23] B. Laurent, P. Cleary, Comparative study by pept and dem for flow and mixing in a ploughshare mixer, Powder Technology 228 (2012) 171-186.

[24] D. Hohner, S. Wirtz, V. Emden, H.K. Scherer, Comparison of the multi-sphere and polyhedral approach to simulate non-spherical particles within the discrete element method, Powder Technology 208 (2011) 643-656.

[25] D. Markauska, Investigation of adequacy of multi-sphere approximation of elliptical particles for DEM simulations, Granular Matter 12 (2010) 107-123.

[26] D. Zhao, E. Nezami, Y. Hashash, J. Ghaboussi.J., Three-dimensional discrete element simulation for granular materials, Computer-Aided Engineering Computations: International Journal for Engineering and Software 23 (2006) 749-770.

[27] M. Pasha, C. Hare, M. Ghadiri, A. Gunadi, P. Piccione, Effect of particle shape on flow in discrete element method simulation of a rotary batch seed coater, Powder Technology 296 (2016) 29-36, (C) 2015, Elsevier. This is an author produced version of a paper published in Powder Technology. Uploaded in accordance with the publisher's self-archiving policy.

URL http://eprints. whiterose.ac.uk/105432/

[28] K. Kildashti, K. Dong, B. Samali, Q. Zheng, A. Yu, Evaluation of contact force models for discrete modelling of ellipsoidal particles, Chemical Engineering Science 177 (2018) 1 - 17.

[29] H. Abou-Chakra, J. Baxter, U. Tuzun, Three-dimensional particle shape descriptors for computer simulation of non-spherical particulate assemblies, Advanced Powder Technology 15 (2004) 63-77.

[30] A. Bar, Superquadrics and angle-preserving transformations, IEEE Computer Graphics and Applications 1 (1981) 11-23.

[31] P. W. Cleary, Particulate mixing in a plough share mixer using dem with realistic shaped particles, Powder Technology 248 (2013) 103 - 120, discrete Element Modelling. doi:https://doi.org/10.1016/j.powtec.2013.06.010.

URL http://www.sciencedirect.com/science/article/pii/S0032591013004166

[32] M. Sinnott, P. Cleary, The effect of particle shape on mixing in a high shear mixer, Computational Particle Mechanics 3 (2016) 477-504. 
[33] A. Radeke, Statistische und mechanische analyse der kraefte und bruchfestigkeit von dicht gepackten granularen medien unter mechanischer belastung, Ph.D. thesis, Freiberg, TU Bergakademie (2006).

[34] N. Govender, D. Wilke, S. Kok, Collision detection of convex polyhedra on the NVIDIA GPU architecture for the discrete element method, Applied Mathematics and Computation 267 (2015) 810-829.

[35] T. A. Satori TSUZUKI, Large-scale agitation simulation based on distinct element method on gpu supercomputer, 2013.

[36] M. Yamamoto, S. Ishihara, J. Kano, Evaluation of particle shape effect for mixing behaviour in a rotating drum by dem simulation, Journal of the Society of Powder Technology, Japan 52 (8) (2015) 445-452. doi:10.4164/sptj.52.445.

[37] S. Pantaleev, S. Yordanova, A. Janda, M. Marigo, J. Y. Ooi, An experimentally validated dem study of powder mixing in a paddle blade mixer, Powder Technology 311 (2017) 287 - 302. doi:https://doi.org/10.1016/j.powtec.2016.12.053.

URL http://www.sciencedirect.com/science/article/pii/S0032591016309305

[38] P. Pizette, Green strength of binder-free ceramic, Journal of the European Ceramic Society 33 (2013) 975-984.

[39] H. Tao, B. Jin, W. Zhong, X. Wang, B. Ren, Y. Zhang, R. Xiao, Discrete element method modeling of nonspherical granular flow in rectangular hopper, Chemical Engineering and Processing: Process Intensification 49 (2010) 151-158.

[40] C. González-Montellano, A. Ramírez, E. Gallego, F. Ayuga, Validation and experimental calibration of 3d discrete element models for the simulation of the dis- charge flow in silos, Chemical Engineering Science 66 (2011) 5116-5126.

[41] C. W. Boon, G. T. Houlsby, S. Utili, A new contact detection algorithm for three-dimensional non-spherical particles, Powder technology 248 (2013) 94-102.

[42] A. V. Potapov, Rocky dem - dem particle simulator, http://www.rocky-dem.com/ (2011-Present).

[43] N. Govender, D. N. Wilke, P. Pizette, N.-E. Abriak, A study of shape non-uniformity and polydispersity in hopper discharge of spherical and polyhedral particle systems using the blaze-dem gpu 
code, Applied Mathematics and Computation 319 (2018) 318 - 336, recent Advances in Computing. doi:https://doi.org/10.1016/j.amc.2017.03.037.

URL http://www.sciencedirect.com/science/article/pii/S0096300317302217

[44] N. Govender, D. Wilke, S. Kok, Blaze-demgpu: Modular high performance dem framework for the gpu architecture, SoftwareX 5 (2016) 62-66.

[45] NVIDIA, Cuda 6 (May 2014).

URL http://www.nvidia.com/cuda

[46] N. Govender, D. Wilke, S. Kok, R. Els, Development of a convex polyhedral discrete element simulation framework for NVIDIA Kepler based GPUs, Journal of Computational and Applied Mathematics 270 (2014) 63-77.

[47] Y. T. Feng, K. Han, D. R. J. Owen, Energy-conserving contact interaction models for arbitrarily shaped discrete elements, Computer Methods in Applied Mechanics and Engineering (2012) 169-177.

[48] R. Venugopal, R. Rajamani, 3d simulation of charge motion in tumbling mills by the discrete element method., Powder Technology 115 (2001) 157-166.

[49] M. Hromnik, Masters Thesis: A GPGPU implementation of the discrete element method applied to modeling the dynamic particulate environment inside a tumbling mill, University of Cape Town, www.uct.ac.za, 2013.

[50] B. Mishra, R. Rajamani, Simulation of charge motion in ball mills. Part 1: experimental verifications, Int. J. Mineral Process 40 (1994) 171-186.

[51] E. Battey-Pratt, T. Racey, Geometric model for fundamental particles, International Journal of Theoretical Physics 19 (1980) 6.

[52] T. Harada, GPU Gems 3: Real-time rigid body simulation on GPUs, Vol. 3, 2008.

[53] N. Govender, R. K. Rajamani, S. Kok, D. N. Wilke, Discrete element simulation of mill charge in 3d using the blaze-dem gpu framework, Minerals Engineering 79 (2015) 152 - 168. doi:https://doi.org/10.1016/j.mineng.2015.05.010.

URL http://www.sciencedirect.com/science/article/pii/S0892687515300042 
[54] N. Govender, P. Pizette, D. Wilke, N. Abriak, Validation of the GPU based Blaze-DEM framework for hopper discharge, Proceedings of the International Conference on Particle-based Methods 2015 Spain, 2015.

[55] C. Radeke, B. Glasser, J. Khinast, Large-scale powder mixer simulations using massively parallel GPU architectures, Chemical Engineering Science 65 (2010) 6435-6442.

[56] D. Koller, A. Posch, G. Hoerl, C. Voura, S. Radl, N. Urbanetz, S. Fraser, W. Tritthart, F. Reiter, M. Schlingmann, J. Khinast, Continuous quantitative monitoring of powder mixing dynamics by near-infrared spectroscopy, Powder Technology 205 (2011) 87-96.

[57] P. Lacey, The mixing of solid particles, Transactions of the Institution of Chemical Engineers 21 (1943) $53-59$.

\begin{tabular}{|c|c|c|c|c|c|}
\hline Particle Shape & Bound Radius $(\mathrm{cm})$ & Volume $\left(\mathrm{cm}^{3}\right)$ & Aspect Ratio & Mass $\left(\mathrm{kg} 10^{-3}\right)$ & SArea $\left(\mathrm{cm}^{2}\right)$ \\
\hline \hline Sphere & $\mathbf{0 . 6 2 0}$ & 0.523 & $\mathbf{1 . 0 0}$ & 1.433 & $\mathbf{3 . 1 4 1}$ \\
\hline Cube & 0.804 & 0.520 & $\mathbf{1 . 0 0}$ & 1.433 & 3.848 \\
\hline TTet & 0.714 & 0.528 & 1.15 & 1.447 & 4.072 \\
\hline Biluna & 0.767 & 0.523 & 1.48 & 1.443 & 3.727 \\
\hline HexPrism & $\mathbf{1 . 2 0 0}$ & 0.524 & $\mathbf{6 . 6 8}$ & 1.442 & $\mathbf{5 . 1 7 2}$ \\
\hline
\end{tabular}

Table 1: Particle geometric properties

\begin{tabular}{|c|c|c|c|}
\hline Particle Type & $\sigma$ & $\bar{\mu}$ & RSD $(\%)$ \\
\hline \hline Sphere & 0.005 & 0.850 & 0.624 \\
\hline Cube & 0.004 & 0.880 & 0.415 \\
\hline TTet & 0.004 & 0.751 & 0.515 \\
\hline Biluna & 0.006 & 0.751 & 0.785 \\
\hline HexPrism & 0.005 & 0.661 & 0.750 \\
\hline Mix & 0.011 & 0.730 & 1.563 \\
\hline
\end{tabular}

Table 2: Relative standard deviation (RSD) of the mixing index of each particle type. RSD is computed from 21 samples, sampled over the last 5 s. 


\begin{tabular}{|c|c|c|c|c|c|c|}
\hline & Sphere & Cube & TTet & Biluna & HexPrism & Mix \\
\hline \hline Sphere & {$[0,2]$} & {$[21,7 \mathrm{E}-23]$} & {$[69,6 \mathrm{E}-43]$} & {$[57,9 \mathrm{E}-40]$} & {$[119,2 \mathrm{E}-52]$} & {$[43,5 \mathrm{E}-35]$} \\
\hline Cubes & & {$[0,2]$} & {$[111,4 \mathrm{E}-51]$} & {$[85,2 \mathrm{E}-46]$} & {$[162,1 \mathrm{E}-57]$} & {$[57,1 \mathrm{E}-39]$} \\
\hline TTet & & & {$[0,2]$} & {$[0.1,1.8]$} & {$[65,5 \mathrm{E}-42]$} & {$[8,3 \mathrm{E}-9]$} \\
\hline Biluna & & & & {$[0,2]$} & {$[53,2 \mathrm{E}-38]$} & {$[7,1 \mathrm{E}-8]$} \\
\hline HexPrism & & & & & {$[0,2]$} & {$[25,5 \mathrm{E}-26]$} \\
\hline Mix & & & & & & {$[0,2]$} \\
\hline
\end{tabular}

Table 3: Two-sample $t$ test for the mixing index results between the various particle pairs, reported as [t-value, p-value] pairs per index.

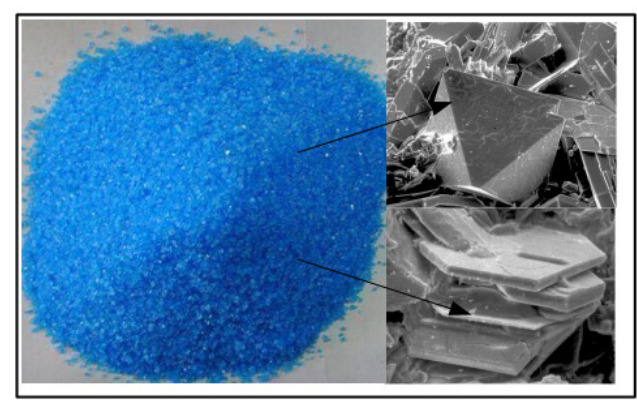

(a)

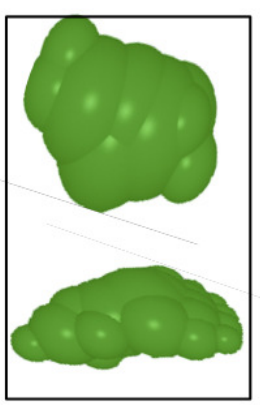

(b)

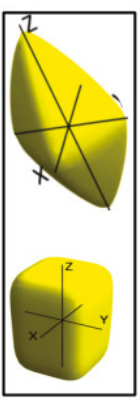

(c)

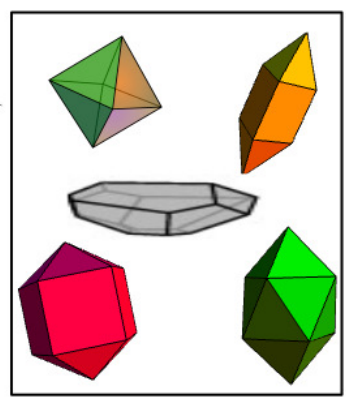

(d)

Figure 1: (a) Typical crystalline powder with SEM scans at the particle scale, with associated DEM approximations using (b) clumped sphere (c) superquadrics and (d) polyhedral representations of particle shape. 


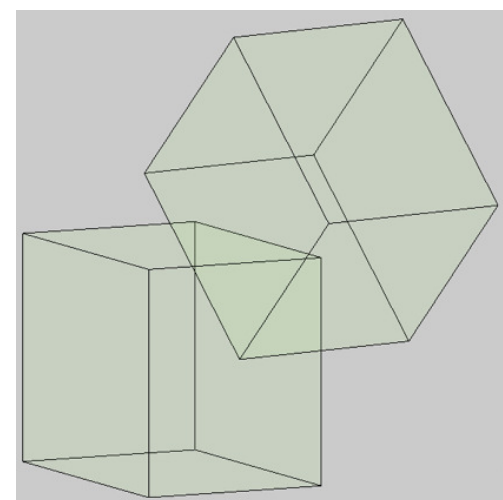

(a)

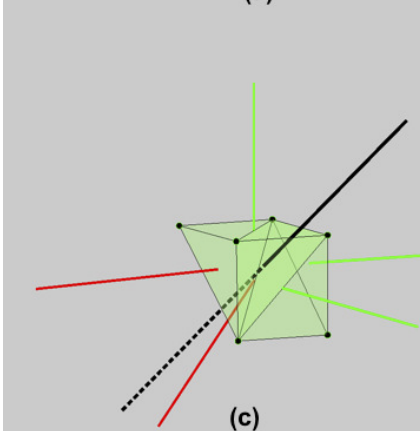

(c)

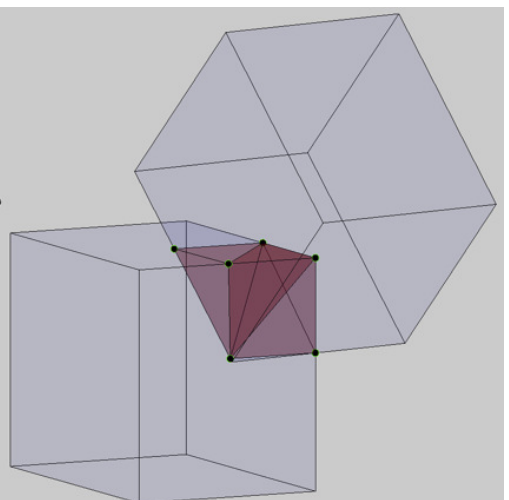

(b)

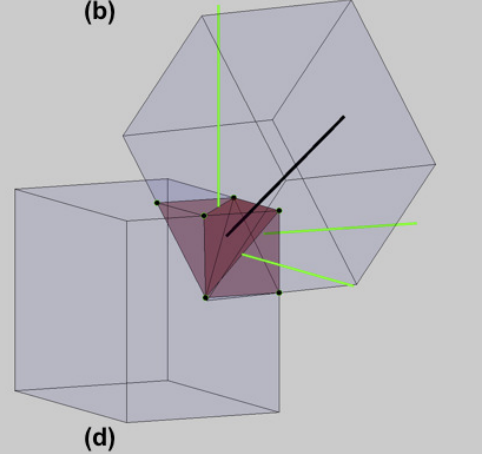

Figure 2: Detail contact model (a) between two intersection polyhedra by resolving the (b) contact points from which the (c) convex hull is constructed to compute the contact volume and contact normal's (red and green lines) with (d) particle forces (dashed and solid black lines) resolved for each particle.

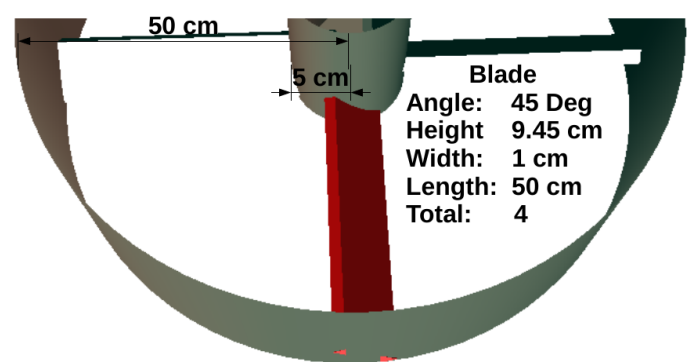

Figure 3: Geometry of the high-shear batch mixer with four blades pitched at 45 degrees rotating inside a stationary vessel. 


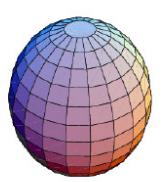

(a)

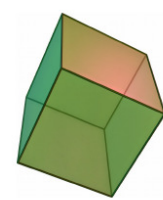

(b)

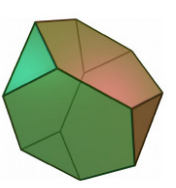

(c)

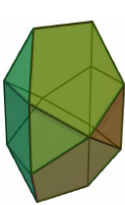

(d)

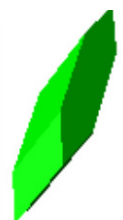

(e)

Figure 4: Particle shapes considered in this study include (a) Cube, (b) Sphere, (d) Truncated Tetrahedron (TTET), (d) Bilunabirotunda (Biluna) and (e) Scaled Hexagonal Prism (HexPrism).

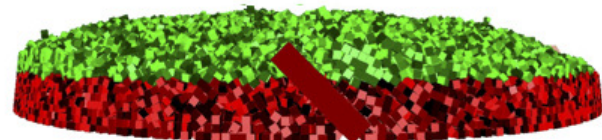

(a)

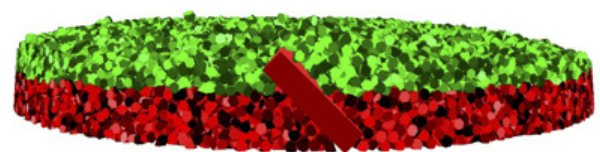

(c)

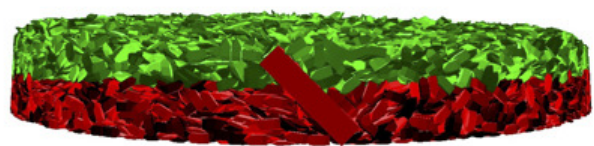

(e)

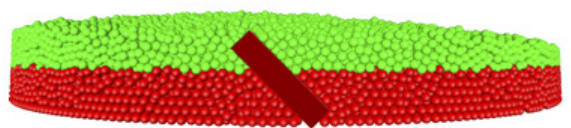

(b)

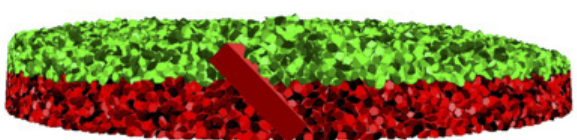

(d)

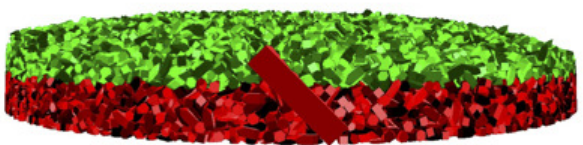

(f)

Figure 5: Initial configuration for the (a) Cube, (b) Sphere, (c) TTet, (d) Biluna, (e) HexPrism, and (f) Mix particle systems. 

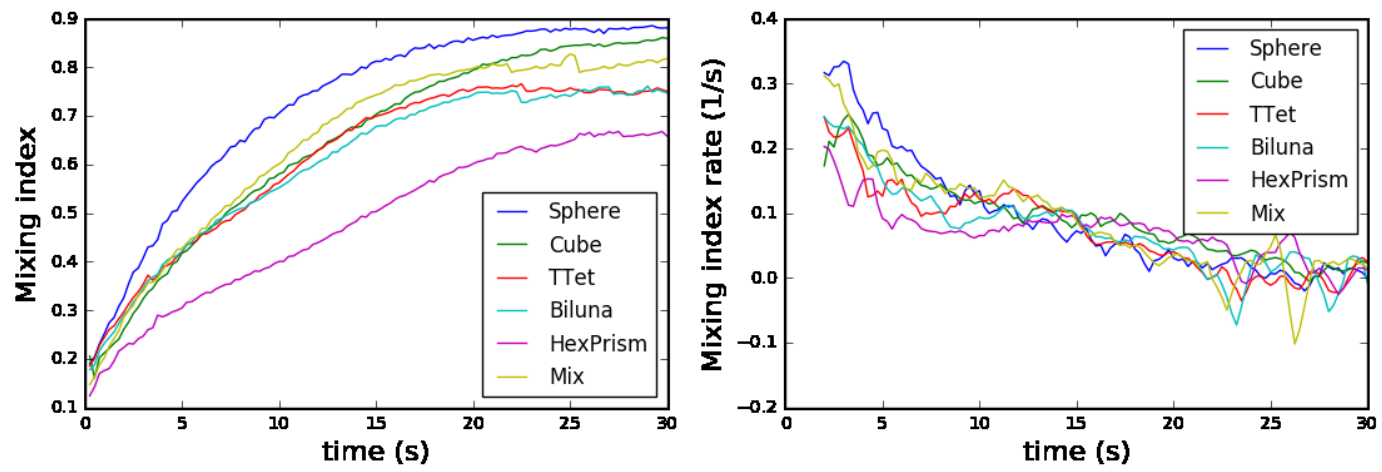

Figure 6: (a) Lacey Mixing Index and (b) Smoothed Lacey Mixing Index Rate for the Cube, Sphere, TTet, Biluna, HexPrism and Mix particle systems over the first 30s of mixing. A 1s windows was used to smooth the Lacey Mixing Index Rate.

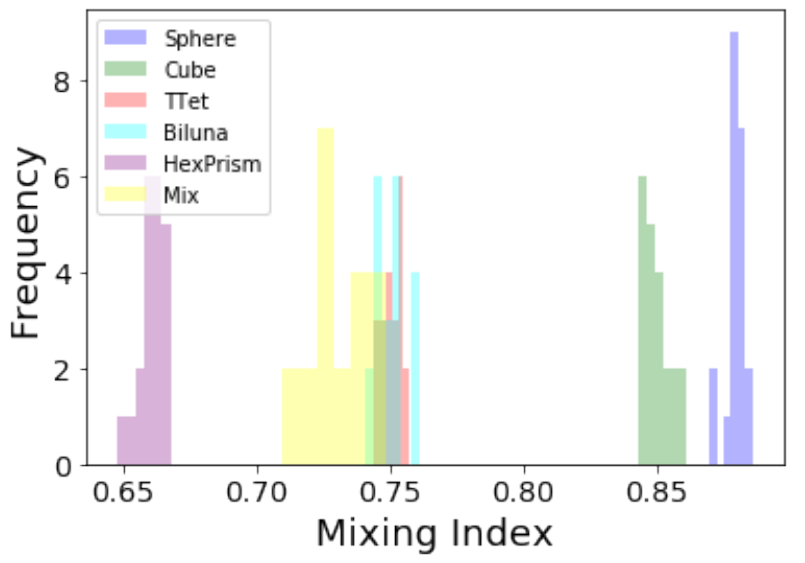

Figure 7: Histogram of the mixing index computed from 21 samples drawn over the last five seconds per particle type. 


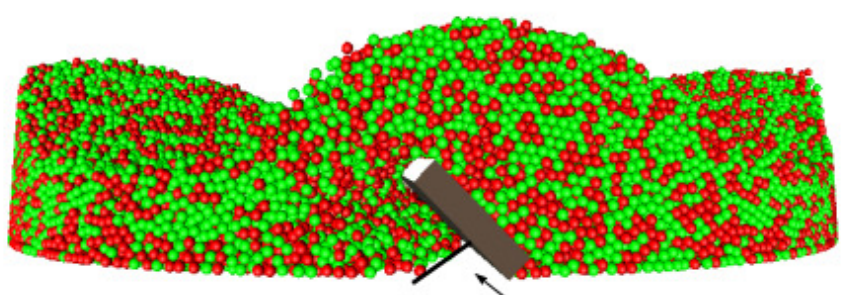

(a)

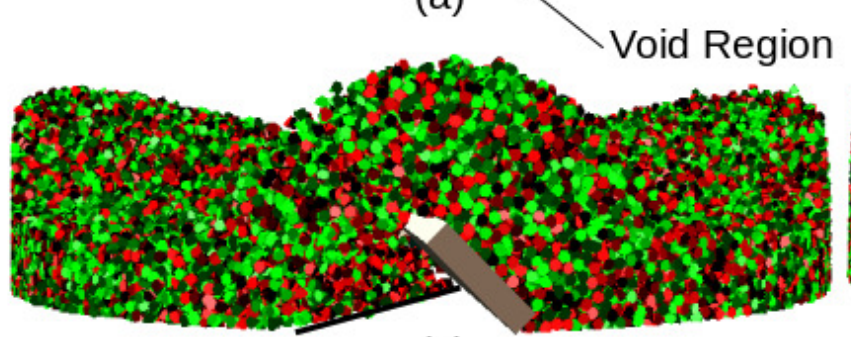

(c)

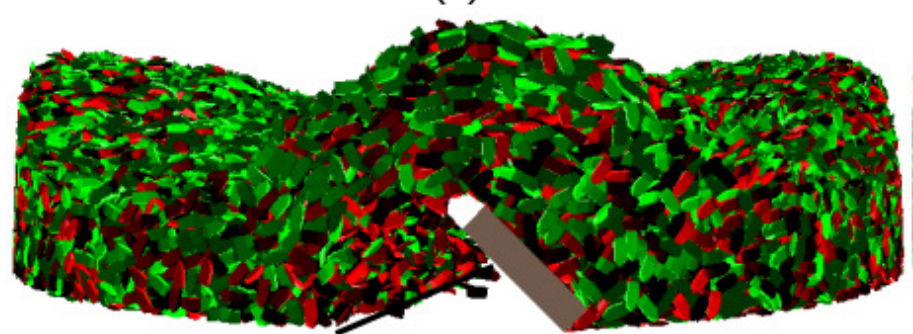

(e)

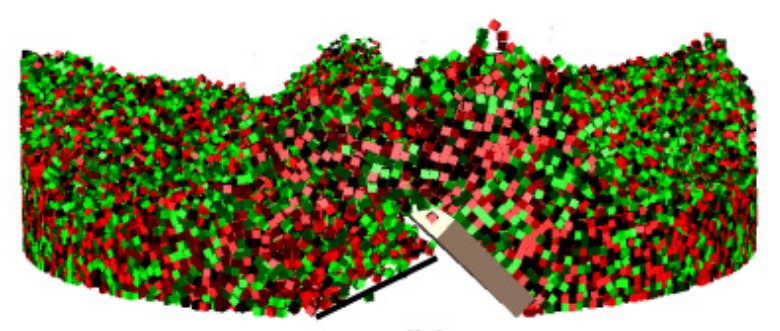

(b)

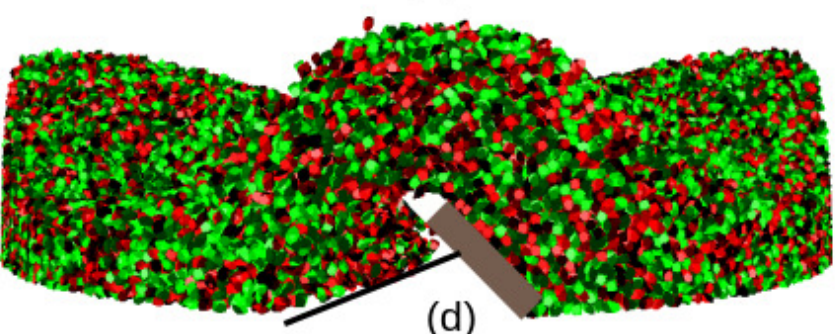

(d)

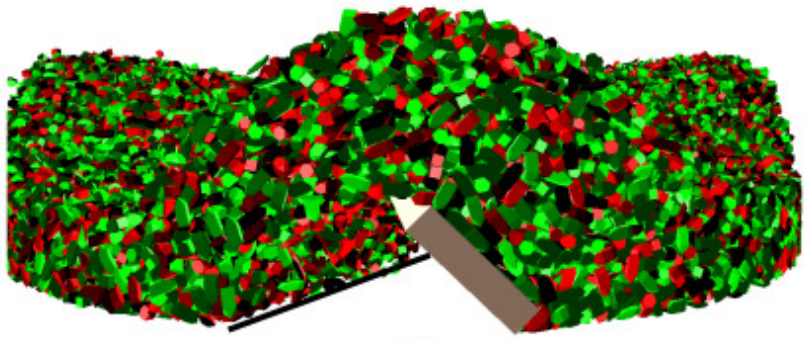

(f)

Figure 8: The mixing state at $\mathrm{t}=30$ s of process time, for the (a) Cube, (b) Sphere, (c) TTet, (d) Biluna, (e) HexPrism and (f) Mix particle systems. 


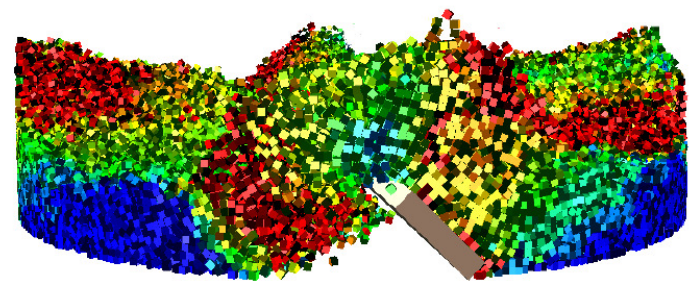

(a)

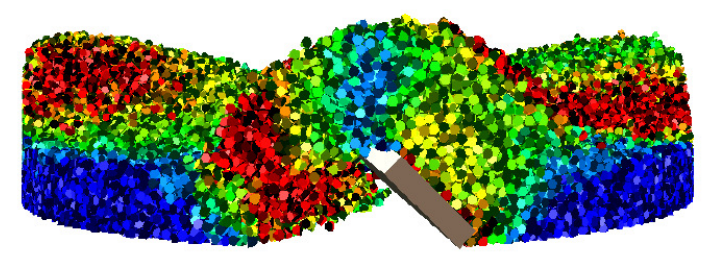

(c)

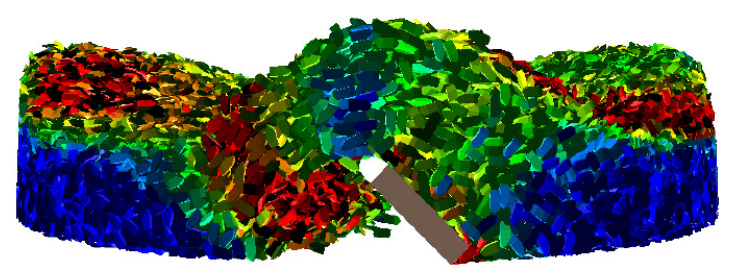

(e)

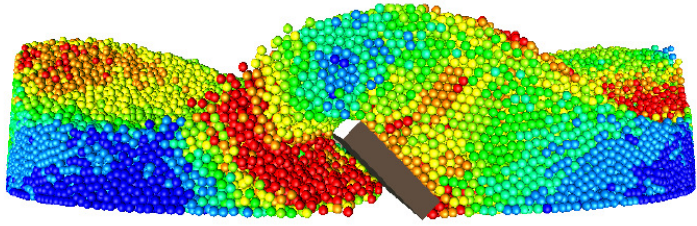

(b)

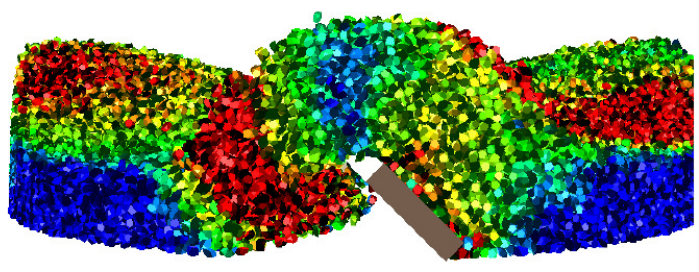

(d)

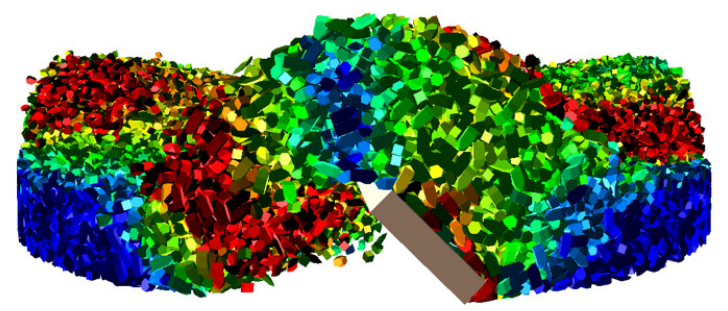

(f)

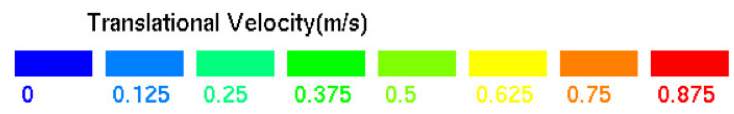

Figure 9: The resultant speed at $\mathrm{t}=30$ s of process time for (a) Cube, (b) Sphere, (c) TTet, (d) Biluna, (e) HexPrism and (f) Mix particle systems. 

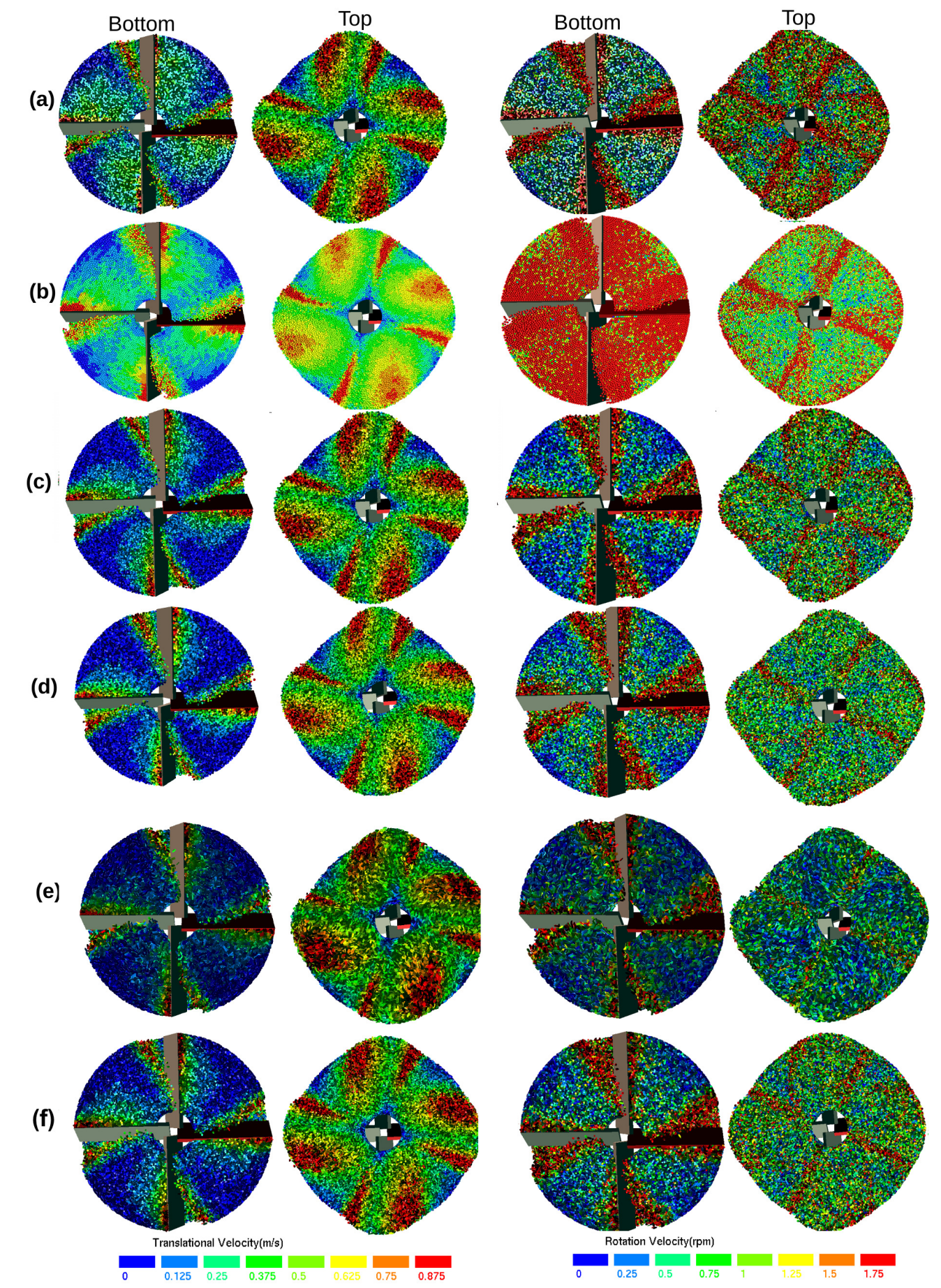

Figure 10: The mixing view at $\mathrm{t}=30 \mathrm{~s}$, the first column resultant speed from the bottom (rotation direction clockwise), second column resultant speed from the top (rotation direction anti-clockwise), third column angular speed from the bottom (rotation direction clockwise) and the fourth column the angular speed from the top (rotation direction anti-clockwise) for the (a) Cube, (b) Sphere, (c) TTet, (d) Biluna, (e) HexPrism and (f) Mix particle systems. 


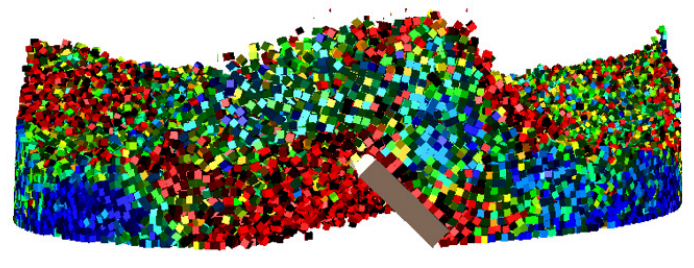

(a)

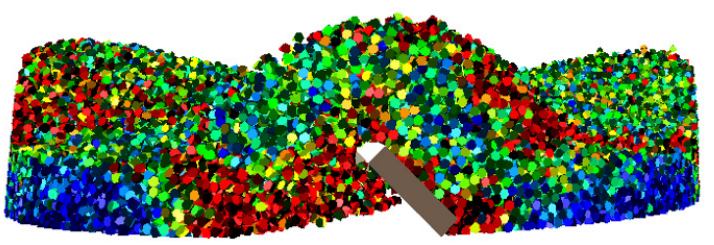

(c)

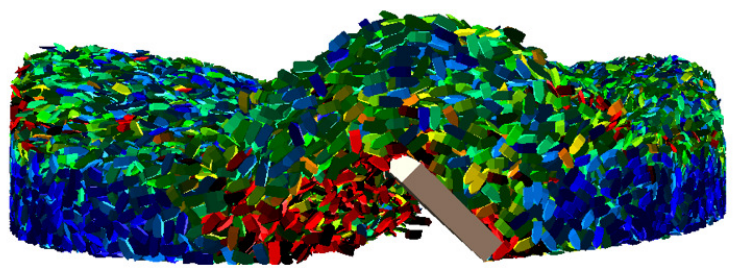

(e)

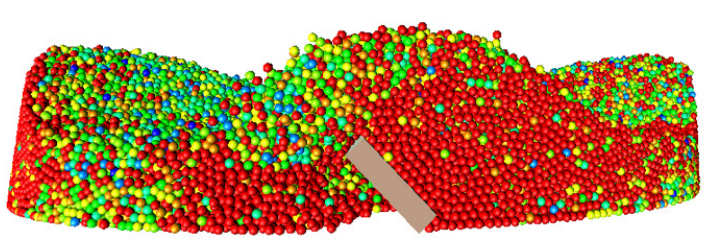

(b)

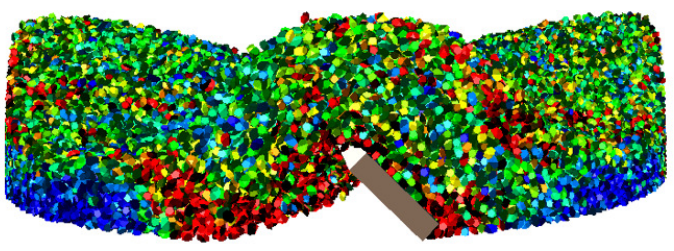

(d)

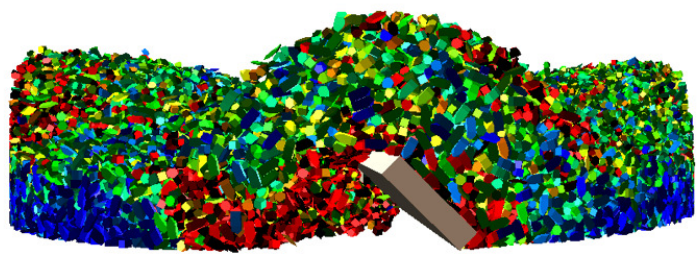

(f)

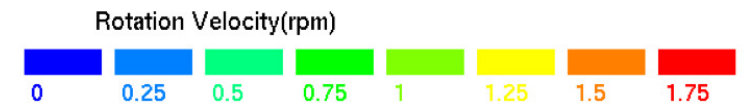

Figure 11: The angular velocity profile at $\mathrm{t}=30$ s of simulation time for (a) Cube, (b) Sphere, (c) TTet, (d) Biluna, (e) HexPrism and (f) Mix particle systems. 

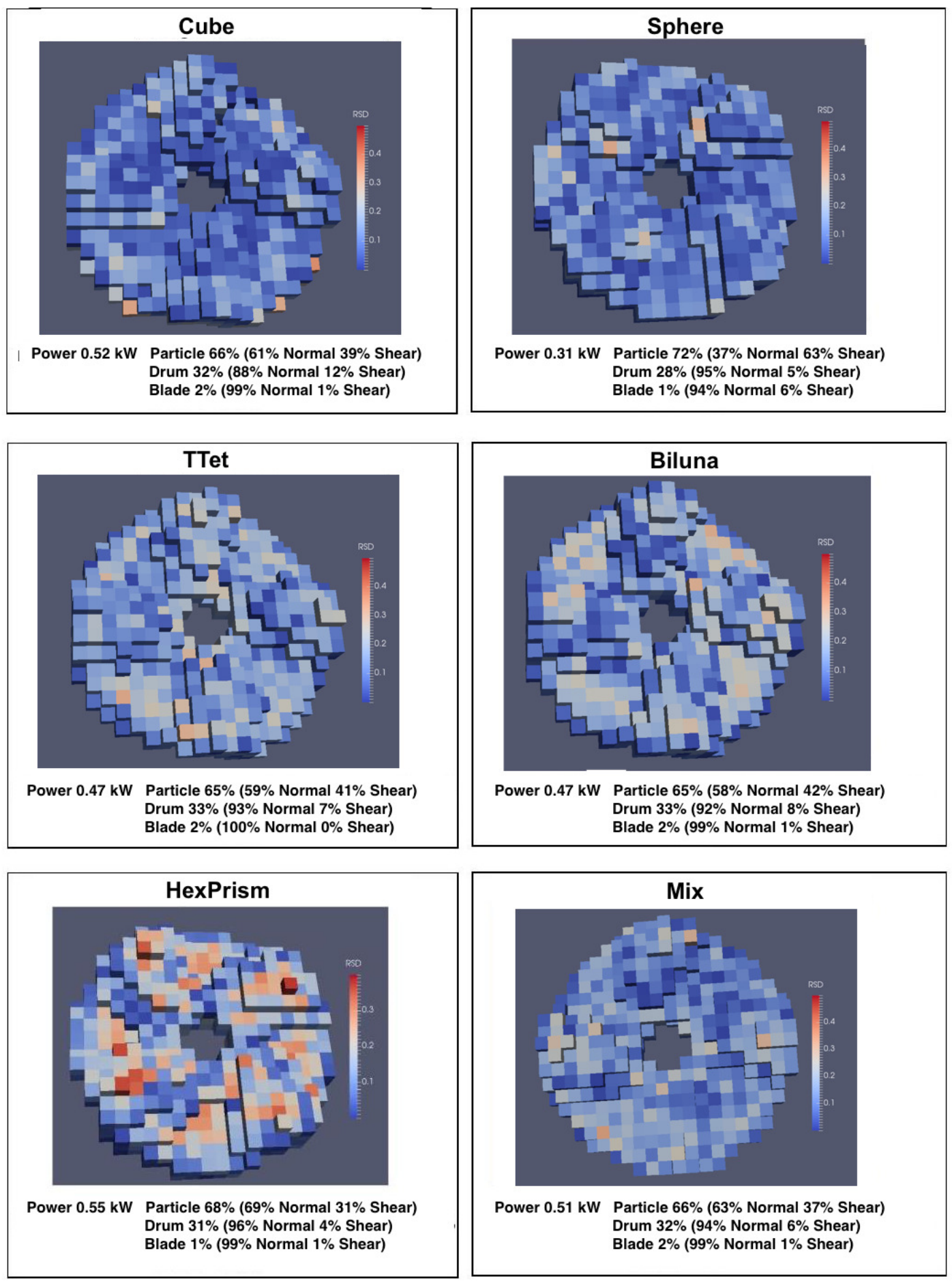

Figure 12: Spatial distribution of RSD at $\mathrm{t}=30 \mathrm{~s}$ and averaged start-up power distribution between particle, blade and drum for the Cube, Sphere, TTet, Biluna, HexPrism and Mix particle systems. 


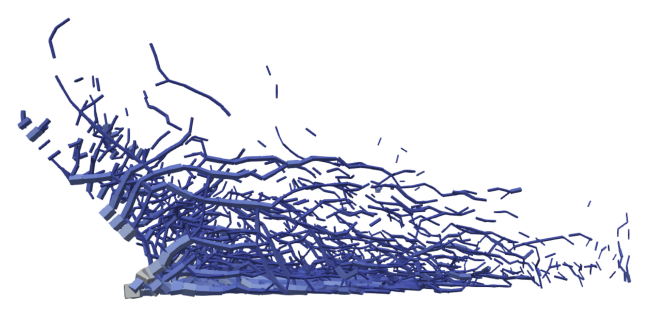

(a)

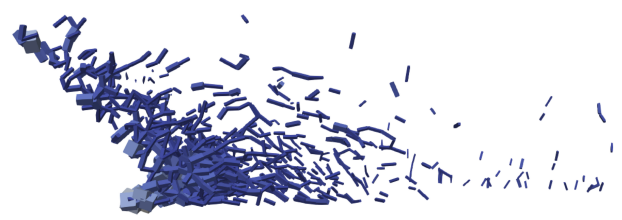

(c)

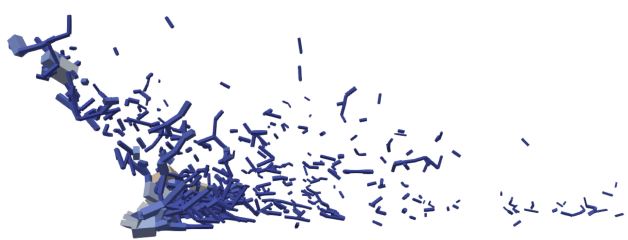

(e)

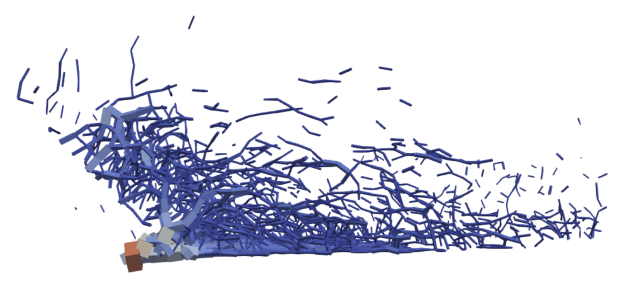

(b)

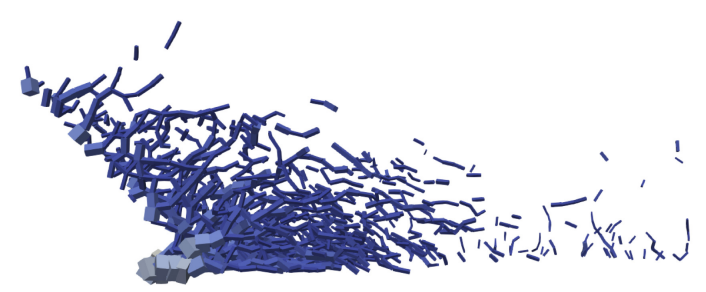

(d)

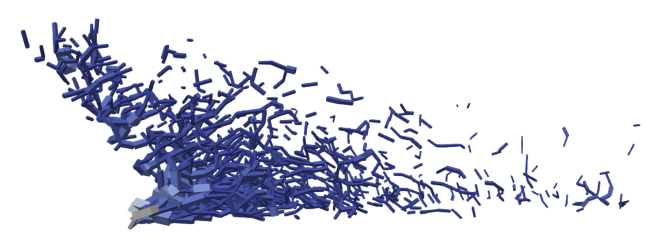

(f)

Figure 13: Inter-particle force chains after 25 seconds for the (a) Cube, (b) Sphere, (c) TTet, (d) Biluna, (e) HexPrism and (f) Mix particle systems. 

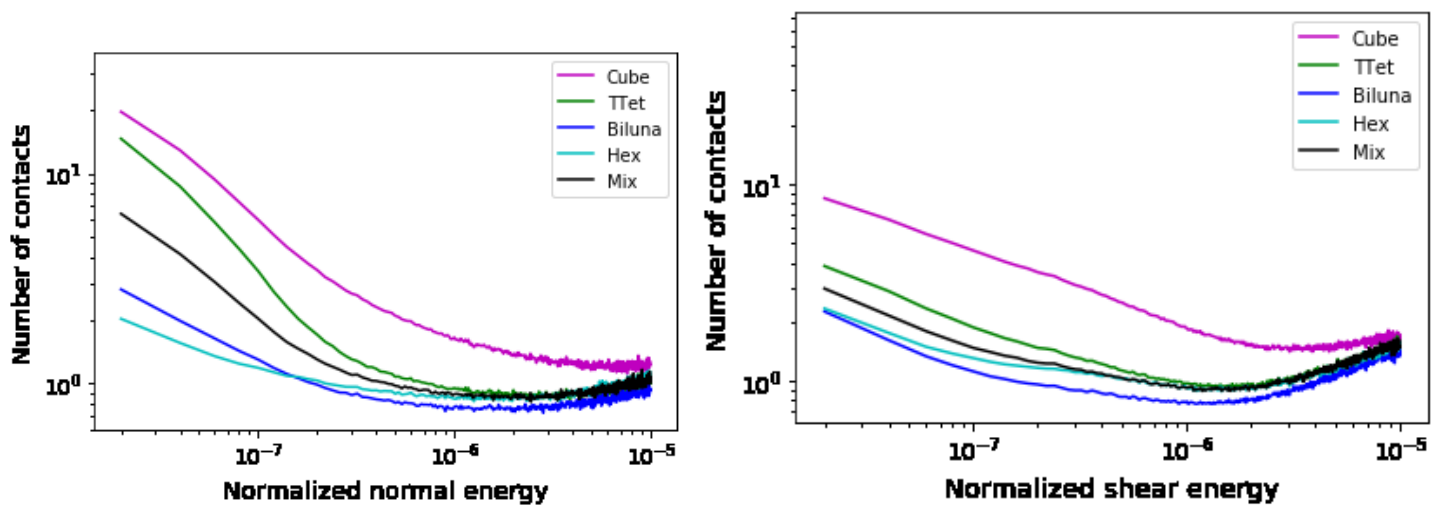

Figure 14: (a) Normal and (b) shear energy spectra.
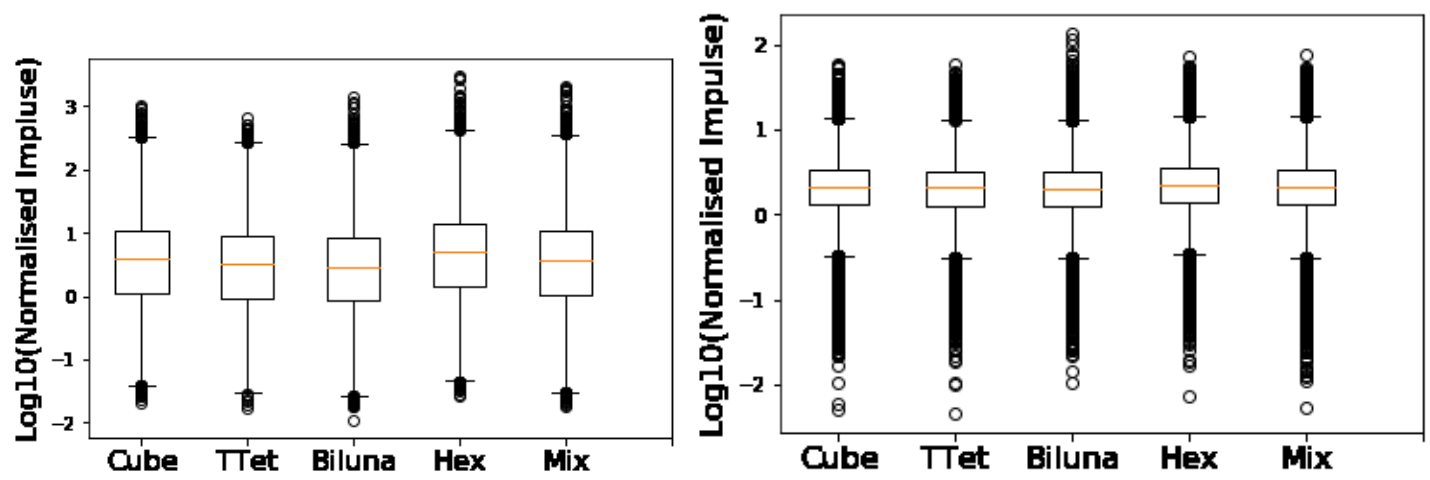

Figure 15: (a) Normal and (b) shear particle impact accumulation. 


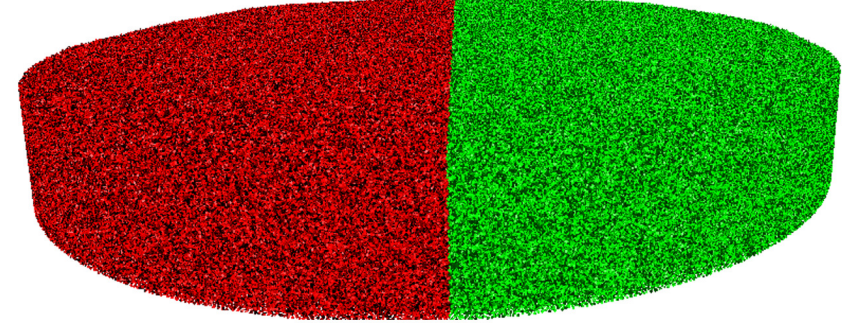

(a)

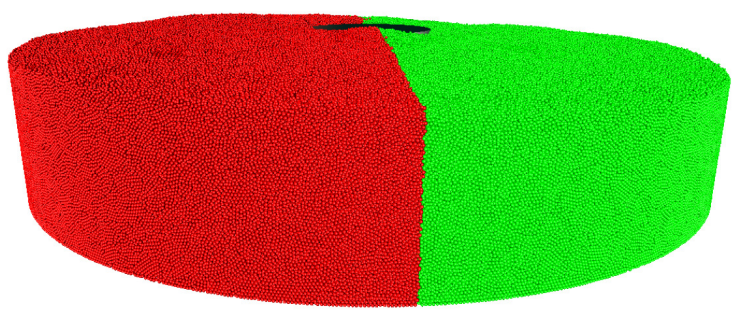

(b)

Figure 16: Initial configuration for (a) a 6 million TTet particle system and (b) a 6 million Sphere particle systems. 


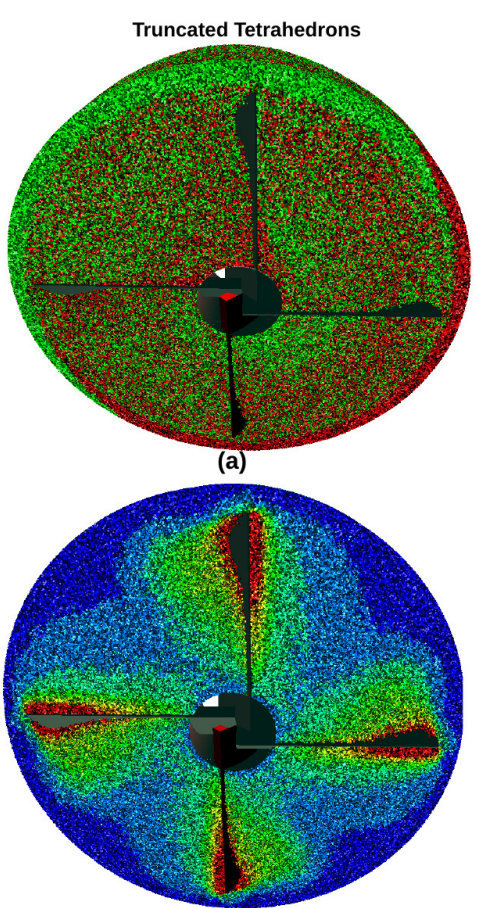

(b)

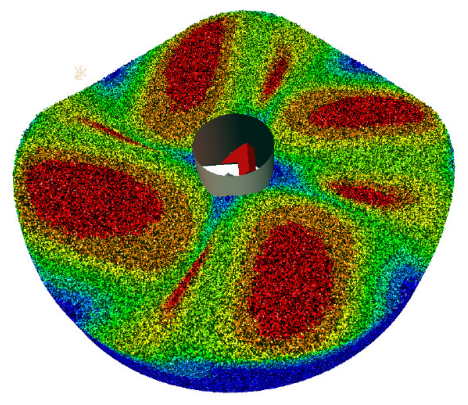

(c)

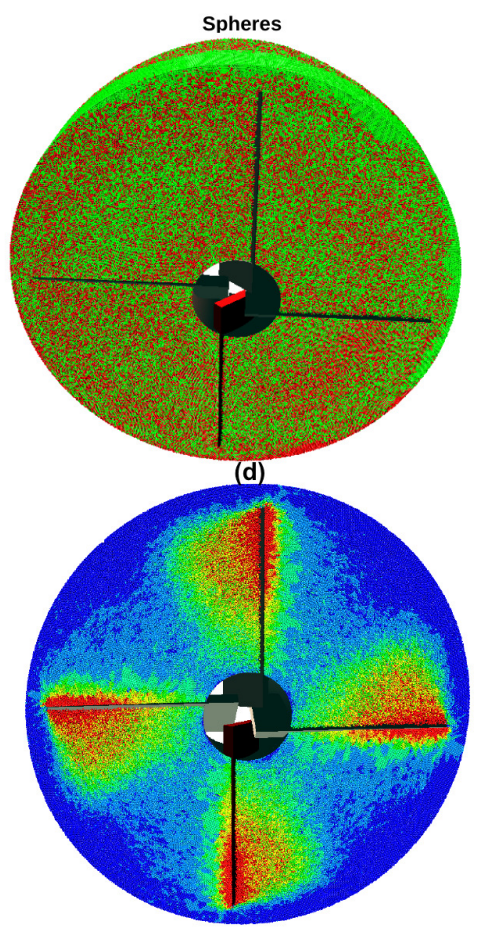

(e)

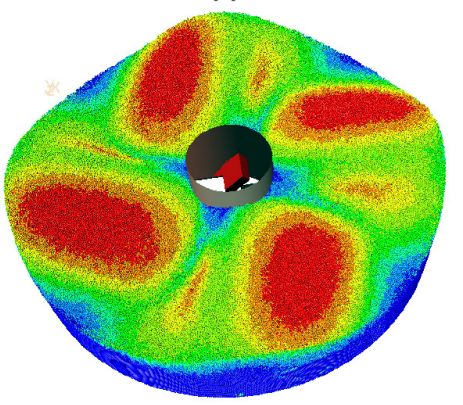

(f)

Figure 17: Semi-industrial scale simulation of a high-shear batch mixer using (a) - (c) 6 million TTet and (d) (f) 6 million Sphere particle system after 30 revolutions $(\mathrm{t}=60 \mathrm{~s})$. 